\title{
Spiral-based chaotic chicken swarm optimization algorithm for parameters identification of photovoltaic models
}

\section{Miao Li}

Information Engineering School of Nanchang University

Chunquan Li ( $\nabla$ lichunquan@ncu.edu.cn)

Nanchang University - Qianhu Campus: Nanchang University

Zhengyu Huang

Information Engineering School of Nanchang University

Jiehui Huang

Information Engineering School of Nanchang University

\section{Gaige Wang}

Ocean University of China

Peter X. Liu

Nanchang University - Qianhu Campus: Nanchang University

\section{Research Article}

Keywords: Chicken swarm optimization algorithm (CSO), spiral shrinkage, parameters identification, photovoltaic models

Posted Date: April 12th, 2021

DOl: https://doi.org/10.21203/rs.3.rs-352988/v1

License: (c) (1) This work is licensed under a Creative Commons Attribution 4.0 International License. Read Full License 


\title{
Spiral-based chaotic chicken swarm optimization algorithm for parameters identification of photovoltaic models
}

\author{
Miao Li ${ }^{1}$, Chunquan Li ${ }^{1}$, Zhengyu Huang ${ }^{1}$, Jiehui Huang ${ }^{1}$, Gaige Wang ${ }^{2}$ and Peter X. Liu ${ }^{1}$
}

\begin{abstract}
Photovoltaic (PV) systems are becoming increasingly significant because they can convert solar energy into electricity. The conversion effeciency is ralated to the PV models' parameters, so it is crucial to identify parameters of PV models. Recently, various heuristic methods have been proposed to identify the parameters, but they cannot provide sufficient accurate and reliable performance. To address this problem, this paper proposes a spiral-based chaos chicken swarm optimization algorithm (SCCSO) including three stratages: i) the information-sharing strategy provides the latest information of the roosters for searching global optimal solution, beneficial to improve the exploitation ability; ii) the spiral motion strategy can enable hens and chicks to move towards their corresponding targets with a spiral trajectory, improving the exploration ability; iii) a self-adaptive-based chaotic disturbance mechanism is introduced around the global optimal solution to generate a promising solution for the worst chick at each iteration, thereby improving the convergence speed of the chicken flock. Besides, SCCSO is used for identifying different PV models such as the single diode, the double diode, and PV module models. Comprehensive analysis and experimental results show that SCCSO provides better robustness and accuracy than other advanced heuristic methods.
\end{abstract}

\section{Keywords}

Chicken swarm optimization algorithm (CSO); spiral shrinkage; parameters identification; photovoltaic models.

\section{Declarations}

\section{Funding}

This work was supported in part by the National Natural Science Foundation of China under Grants 61863028, 81660299, and 61503177, and in part by the Science and Technology Department of Jiangxi Province of China under Grants 20161ACB21007, 20171BBE50071, and 20171BAB202033.

\section{Conflicts of interest/Competing interests}

The authors declare that they have no known competing financial interests or personal relationships that could have appeared to influence the work reported in this paper.

\section{Availability of data and material}

All data generated or analysed during this study are included in this published article.

\section{Code availability}

Not applicable.

\section{Authors' contributions}

Miao Li: Methodology, Software, Formal analysis, Writing - original draft. Chunquan Li: Funding acquisition, Writing - review \& editing, Supervision. Zhengyu Huang: Methodology, Validation, Writing - review \& editing. Jiehui Huang: Conceptualization, Writing - review \& editing. Gaige Wang: Writing - review \& editing, Supervision. Peter X. Liu: Writing - review \& editing, Supervision.

\section{Ethics approval}

Not applicable.

Consent to participate

Not applicable.

Consent for publication

Written informed consent for publication was obtained from all participants.

\footnotetext{
Chunquan Li

lichunquan@ncu.edu.cn

Information Engineering School, Nanchang University, Nanchang 330031, China

Department of Computer Science and Technology, Ocean University, Qingdao 266100, China
} 
With the depletion of non-renewable energy, it is more and more urgent to find clean alternative energy, and thus people pay more attention to the utilization of renewable energy (Qais et al. 2019a). Among all renewable energy sources, solar energy is regarded as inexhaustible clean energy, which has attracted worldwide attention due to its low development cost and huge development potential (Manel et al. 2018). Solar energy can be converted into electricity through PV systems (Zhang et al. 2020b). Nevertheless, the utilization efficiency of solar energy is greatly affected by weather and other environmental factors as PV arrays of PV systems are easily damaged in those tough conditions (Eseye et al. 2017; Zhang et al. 2020a). As a result, to control and optimize PV systems, it is significant to build accurate PV models by measured voltage-current data for evaluating the actual operation behavior of PV arrays (Li et al. 2020; Zaimi et al. 2019).

Several mathematical models have successfully depicted the performance and nonlinear behavior of PV systems. Among them, the single diode model and double diode model are widely adopted in various practical problems (Aly et al. 2019; Liu et al. 2020). What's more, the accuracy of PV models mainly depends on their parameters. However, the parameters usually change and even are unavailable owing to aging, faults, and unstable operating conditions of the PV systems (Harrou et al. 2018; Jiao et al. 2020). Therefore, to effectively simulate, evaluate, and control PV systems, it is indispensable to identify the model parameters accurately and reliably. This has triggered the upsurge of researchers in developing various optimization methods for the parameter identification of PV models.

In essence, the parameter identification of PV models can be regarded as an optimization problem and solved by building an effective objective function. To obtain the corresponding optimal solutions, various optimal algorithms have been designed. They can be roughly divided into deterministic methods and heuristic methods (Abdel-Basset et al. 2021a). The deterministic methods need to be combined with several model constraints, including differentiability and convexity (Elsheikh et al. 2019). It is apt for the deterministic methods to fall into the local optimum and their performance largely depends on their initial solutions (Gao et al. 2018; Ridha et al. 2020). In contrast, the performance of the heuristic methods is almost not affected by model constraints (Jordehi 2018), so they are used to solve various parameters identification of PV models.

Many heuristic algorithms have already been put in use. Niu et al. proposed an improved TLBO (ITLBO) with elite strategy to obtain parameters of PV models according to the current-voltage results of solar cells (Niu et al. 2014). Chen et al. developed a generalized opposite teaching learning-based optimization algorithm (GOTLBO) to solve two parameter identification problems of solar cell models, including single diode model and double diode model (Chen et al. 2016). Yu et al. introduced the self-adaptive teaching-learning-based optimization algorithm (SATLBO) to accurately and reliably identify the PV model parameters (Yu et al. 2017a). Abdel-Basset et al. applied a modified teaching-learning based optimization (MTLBO) to the accurate and efficient parameter estimation of PV models (Abdel-Basset et al. 2021b). Jaya algorithm proposed by Rao can perform effective exploration and was widely used to identify the parameters of PV models (Venkata Rao 2016). Additionally, other Jaya based algorithms are also widely utilized in PV models' parameter identification. For instance, the new Jaya algorithm based on elite opposition (EOJaya) (A et al.), an improved JAYA optimization algorithm (IJAYA) (Yu et al. 2017b), and the performance-guided JAYA (PGJAYA) algorithm (Yu et al. 2019). Yu et al. designed a multiple learning backtracking search algorithm (MLBSA) for the parameters identification of different PV models (Yu et al. 2018). Xiong et al. proposed a modified search strategy-assisted crossover whale optimization algorithm (MCSWOA) to extract accurate parameters of PV models (Xiong et al. 2019). Allam et al. used the moth flame optimization algorithm (MFO) for the parameter extraction process of a three diode model, a double diode and the modified double diode models of the same cell/module (Allam et al. 2016). An improved cuckoo search optimization is presented for the parameters extraction of PV cells (Gude and Jana 2020), and a teaching-learning-based artificial bee colony (TLABC) is employed for the solar PV parameters estimation problems (Chen et al. 2018). Besides, an improved brain storming optimization algorithm (IBSO) (Yan et al. 2019), and hybrid symbiotic differential evolution moth-flame optimization algorithm (HSDEMFO) are also presented to accurately identify parameters of PV models (Wu et al. 2020). However, as the parameters identification problem of PV models is multimodal, nonlinear, and contains lots of local optimums, most heuristic algorithms are not easy to sink into global optimal solutions. Therefore, it is still a challenging task to develop a competitive algorithm for identifying the parameters of different PV models accurately and reliably.

Inspired by the foraging behavior and hierarchical order of chickens in nature, Meng et al. proposed a biological heuristic algorithm in 2014, namely chicken swarm optimization algorithm (CSO), which is employed to solve lots of optimization problems in the real world (Meng et al. 2014). In CSO, the information in the chicken flock can be fully utilized to achieve the balance between the exploration ability and the exploitation ability. Moreover, CSO can obtain better results than some classical swarm intelligence optimization algorithms such as PSO, DE, and BA when solving 12 benchmark function problems and other practical engineering problems (Meng et al. 2014). Owing to its advantage in solving optimization problems, CSO has been applied in collaborative beamforming in wireless sensor network (Al Shayokh and Shin 2017), evaluation of regional water resources carrying capacity (Yu et al. 2020), robot path planning (Mu et al. 2016), fault detection issues (Moldovan et al. 2018), and other issues (Ahmed et al. 2018; Sanchari et al. 2018; Taie et al. 2017).

Nevertheless, CSO has some disadvantages in solving the parameters identification problem of PV models such as poor ability in jumping out from the local optimum, insufficient information utilization, and low convergence speed. To overcome these mentioned shortcomings, this paper proposes a spiral-based chaotic chicken swarm optimization algorithm (SCCSO), which involves an information-sharing strategy, a spiral motion strategy, and a self-adaptive-based chaotic disturbance mechanism. First, an information-sharing strategy is employed for roosters to fully use their latest information about food. Two roosters are randomly selected before the update of each rooster. When updating, each rooster draws near the rooster with a better fitness value while keeps away from the other rooster with a worse fitness value, which is propitious to improve the exploitation ability. Second, the spiral motion strategy allows both hens and chicks to move to their respective targets in a spiral trajectory, which expands the 
searching range and improves the exploration ability. Especially, to fully use the information in the chicken flock, chicks follow roosters in early iteration stages while coming after hens in the later iteration stages. Third, a self-adaptive-based chaotic disturbance mechanism is introduced to improve the convergence speed. By imposing a certain disturbance on the global optimal solution, a new individual is generated to update the individual with the worst fitness value, which effectively improves the convergence speed. To verify the accuracy and robustness of SCCSO, it is compared with other advanced algorithms on different PV models including single diode, double diode, and PV module models. Experimental results show that the proposed SCCSO provides better robustness and accuracy compared with other advanced heuristic algorithms.

The main contributions of this paper are given as follows.

- A new algorithm SCCSO is proposed to solve the parameters identification problems of PV models.

- An information-sharing strategy is designed to search for a globally optimal solution by the latest information in roosters and improve the exploitation ability.

- A spiral motion strategy is applied to expand the scope of searching global optimal solutions and improve the exploration ability.

- A self-adaptive-based chaotic disturbance mechanism is employed by guiding the worst chick move near the global optimal solution, speeding up the convergence speed of the chicken flock.

- The effectiveness of SCCSO is comprehensively evaluated in the parameters identification problems of various PV models.

Following is the structure of the rest paper. Section 2 shows the modeling of PV systems and the problem formulation. Section 3 briefly introduces the original CSO. The proposed SCCSO algorithm is introduced detailedly in Section 4. Section 5 analyzes and compares the experimental results. Finally, Section 6 concludes this paper.

\section{Modeling and problem formulation}

Several PV models have been developed to describe the current-voltage characteristics of solar cells in the literature. Only the single diode model and double diode model have been put into factual optimization problems. Brief descriptions and objective functions of these models are presented in this section.

\subsection{Single diode model}

The equivalent circuit of the single diode model is shown in Fig. $1^{3}$.

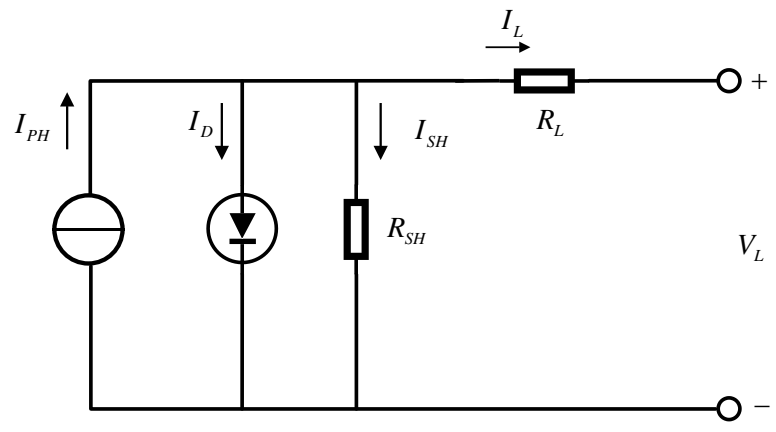

Fig. 1 Equivalent circuit of the single diode model

As we can see, it is composed by a current source connected in parallel with the diode, a shunt resistor representing the leakage current passing through, and a series resistor of the load current considering the related loss. According to Kirchhoff's current law (Diantoro et al. 2018), the output current is calculated by (1).

$$
I_{L}=I_{P H}-I_{D}-I_{S H}
$$

where $I_{L}$ is the output current, and $I_{P H}$ is the photo-generated current. $R_{S H}$ and $R_{L}$ are the series resistance. $I_{D}$ is the current through the diode obtained by Shockley diode [41], and $I_{S H}$ is the current through the shunt resistor. Both of them are calculated by (2) and (3), respectively.

$$
\begin{gathered}
I_{D}=I_{S D}\left[\exp \left(\frac{V_{L}+R_{L} I_{L}}{n V_{t}}\right)-1\right] \\
I_{S H}=\frac{V_{L}+R_{L} I_{L}}{R_{S H}}
\end{gathered}
$$

where $I_{S D}$ is the reverse saturation current, $V_{L}$ is the output voltage of the port, $n$ is the ideal factor of the diode, and $V_{t}$ is the Thermal voltage calculated by (4).

$$
V_{t}=\frac{K t}{q}
$$

where $K$ is Boltzmann constant $\left(1.3806503 \times 10^{-23} \mathrm{~J} / K\right), t$ is the working temperature of the diode in Kelvin. $q$ is the charge amount of meta charge $\left(1.60217646 \times 10^{-19} \mathrm{C}\right)$. By combining (1)-(4), (5) is obtained to reflect the relationship between the output current, the output voltage, and the PV model parameters.

\footnotetext{
3 The figure was drawn with Visio.
} 


$$
I_{L}=I_{P H}-I_{S D}\left[\exp \left(\frac{\left(V_{L}+R_{L} I_{L}\right) q}{n K t}\right)-1\right]-\frac{V_{L}+R_{L} I_{L}}{R_{S H}}
$$

From (5), it can be seen that five unknown parameters $\left(I_{P H}, I_{S D}, R_{L}, R_{S H}\right.$, and $\left.n\right)$ need identifying for the single diode model. The more accurate these parameters are identified, the better the characteristics of PV systems can be reflected.

\subsection{Double diode model}

Fig. $2^{3}$ shows the equivalent circuit of the double diode model.

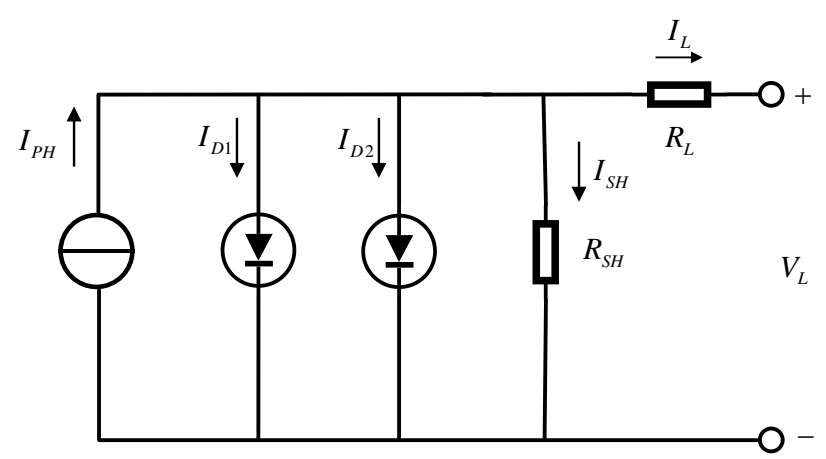

Fig. 2 Equivalent circuit of the double diode model

As we can see, it is formed by connecting a diode in parallel at both ends of the diode connected in parallel with the shunt resistor and current source. By combining (2) and (4), the output current can be calculated as

$$
I_{L}=I_{P H}-I_{S D 1}\left[\exp \left(\frac{V_{L}+R_{L} I_{L}}{n_{1} V_{t}}\right)-1\right]-I_{S D 2}\left[\exp \left(\frac{V_{L}+R_{L} I_{L}}{n_{2} V_{t}}\right)-1\right]-\frac{V_{L}+R_{L} I_{L}}{R_{S H}}
$$

where $I_{D 1}$ and $I_{D 2}$ are diffusion current and saturation current, respectively. $I_{S D 1}$ and $I_{S D 2}$ are reverse saturation current of rectifier diode and compound diode, respectively. $n_{1}$ and $n_{2}$ are the ideal factor of two diodes. From (6), there are seven unknown parameters $\left(I_{P H}, I_{S D 1}, I_{S D 2}, \quad R_{L}, R_{S H}, n_{1}\right.$, and $\left.n_{2}\right)$ need identifying for the double diode model.

\subsection{Photovoltaic module model}

Fig. $3^{3}$ provides the equivalent circuit of a single diode PV module, which is composed of a plurality of solar cells connected in parallel and series.

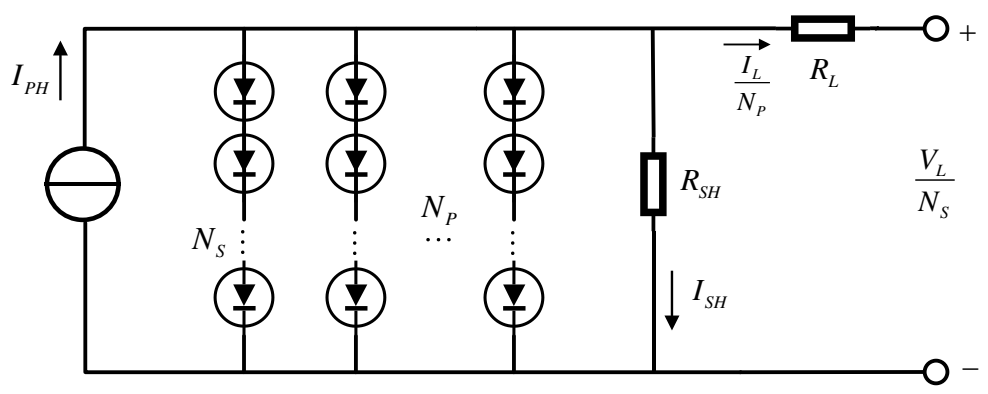

Fig. 3 Equivalent circuit of the PV module model

The output current $I_{L}$ is calculated by (7).

$$
\frac{I_{L}}{N_{P}}=I_{P H}-I_{S D}\left[\exp \left(\frac{\left(V_{L} \cdot N_{P}+R_{L} I_{L} \cdot N_{S}\right) q}{n K t \cdot N_{S} N_{P}}\right)-1\right]-\frac{V_{L} \cdot N_{P}+R_{L} I_{L} \cdot N_{S}}{R_{S H} \cdot N_{S} N_{P}}
$$

where $N_{P}$ and $N_{S}$ represent the number of solar cells connected in parallel and series, respectively. Here, the unknown parameters to be identified are $I_{P H}, I_{S D}, R_{L}, R_{S H}$, and $n$.

\subsection{Objective function}

The PV models' parameters identification problem can be transformed into optimization problems, minimizing the difference between the experimental and simulated data. The error functions of the single and double diode models are respectively defined by (8) and (9):

$$
\begin{gathered}
\left\{\begin{array}{c}
F_{\text {error }}\left(V_{L}, I_{L}, X\right)=I_{P H}-I_{S D}\left[\exp \left(\frac{\left(V_{L}+R_{L} I_{L}\right) q}{n K t}\right)-1\right] \\
\quad-\frac{V_{L}+R_{L} I_{L}}{R_{S H}}-I_{L} \\
X=\left\{I_{P H}, I_{S D}, R_{L}, R_{S H}, n\right\}
\end{array}\right. \\
\left\{\begin{array}{c}
F_{\text {error }}\left(V_{L}, I_{L}, X\right)=I_{P H}-I_{S D 1}\left[\exp \left(\frac{\left(V_{L}+R_{L} I_{L}\right) q}{n_{1} K t}\right)-1\right] \\
-I_{S D 2}\left[\exp \left(\frac{\left(V_{L}+R_{L} I_{L}\right) q}{n_{2} K t}\right)-1\right]-\frac{V_{L}+R_{L} I_{L}}{R_{S H}}-I_{L} \\
X=\left\{I_{P H}, I_{S D 1}, I_{S D 2}, R_{L}, R_{S H}, n_{1}, n_{2}\right\}
\end{array}\right.
\end{gathered}
$$


The root mean square error (RMSE) (Qais et al. 2020a) is used as the objective function of the overall difference between experimental and simulated current data:

$$
\operatorname{RMSE}(X)=\sqrt{\frac{1}{N_{m}} \sum_{i=1}^{N} F^{i} \text { error }\left(V_{L}, I_{L}, X\right)^{2}}
$$

where $X$ is the solution vector composed of the PV model parameters to be identified, and $N_{m}$ is the number of measured $I-V$ data pairs, $i \in\{1,2, \cdots, N\}$.

\section{Original CSO algorithm}

In 2014, inspired by the foraging behavior and hierarchy of the chicken flock in nature, Meng et al. proposed a chicken swarm optimization algorithm (CSO), which is applied to solve various optimization problems (Meng et al. 2014). For simplicity, the behavior of the chicken flock is idealized by the following regulations:

(1) The chicken flock is divided into rooster sub-flock, hen sub-flock, and chick sub-flock according to the fitness value of each individual in the chicken flock. The individuals with better fitness values are defined as roosters, followed by hens, and those with the worst fitness values act as chicks. Particularly, the individual with the best fitness value is defined as the leader rooster, and some of the hens are randomly selected as mother hens. The mother-child relationship is established by randomly choosing motherhens and chicks. Similarly, the spouse relationship between hens and roosters is also done by randomly selecting hens and roosters.

(2) The entire chicken flock is divided into several clusters. Each cluster consists of a rooster, some hens, and some chicks.

(3) The identity of each individual is updated according to the corresponding fitness value at every $\mathcal{G}$ generation. When the identity of each individual is updated, the mother-child and the spouse relationship are also updated.

(4) The individuals in each sub-flock search for food under the leadership of roosters and the food is defined as the global optimal solution. Specifically, hens follow their spouses for food and steal food from other hens or roosters. Chicks follow their corresponding mother-hens to forage. The better the fitness values of individuals are, the more dominant the individuals are in the process of foraging or stealing food.

In CSO, the position of each individual is regarded as a candidate solution for optimization problems. $\mathcal{N}$ is the number of individuals in the chicken flock. Each individual searches food in $\mathcal{D}$ space with dimensions and update its identity at every $\mathcal{G}$ generation. The range of the serial numbers of each individual is $\left\{1,2,3, \cdots, N_{r}, N_{r}+1, \cdots, N_{h}, N_{h}+1, \cdots, N_{c}\right\}$, where $N_{r}, N_{h}$, and $N_{c}$ are the maximum serial numbers of the roosters, hens, and chicks in each sub-flock after sorting, respectively. More details are shown as follows.

\subsection{Rooster foraging}

Each rooster forages under the guidance of other roosters in the rooster sub-flock. The original position and the updated position of each rooster are defined respectively as $X_{i, j}$, and $X_{i, j}^{\text {new }}$, where $i \in\left\{1,2,3, \cdots, N_{r}\right\}, j \in\{1,2,3, \cdots, D\}$. Roosters with smaller fitness can forage food in a wider searching range. The foraging behaviors are expressed by (11) and (12), respectively.

$$
\begin{aligned}
X_{i, j}^{\text {new }} & =X_{i, j} \cdot\left(1+\Re \operatorname{Randn}\left(0, \sigma^{2}\right)\right) \\
\sigma^{2} & =\left\{\begin{array}{l}
\exp \left(\frac{\left(f_{n}-f_{i}\right)}{\left|f_{i}\right|+\zeta}\right), \text { if } f_{i}>f_{n} \\
1, \text { otherwise }
\end{array}\right.
\end{aligned}
$$

where $\Re a n d n\left(0, \sigma^{2}\right)$ is the normal distribution with the average value of 0 and the standard deviation of $\sigma^{2} . f$ is the fitness function. $f_{i}$ and $f_{n}$ are the fitness values of the $i$ th rooster and the $n$th rooster, respectively, where $i, n \in\left\{1,2,3, \cdots, N_{r}\right\}$, and $i \neq n . \zeta$ is a number approaching 0 , which is used to prevent the denominator $\left|f_{i}\right|+\zeta$ from being 0 .

\subsection{Hens foraging}

In the chicken flock, hens can obtain food by following their spouses or stealing from other roosters and hens. The original position and the updated position of each hen are defined respectively as $X_{i, j}$ and $X_{i, j}^{\text {new }}$, where $i \in\left\{N_{r}+1, N_{r}+2, \cdots, N_{h}\right\}$, $j \in\{1,2,3, \cdots, D\} . X_{c, j}$ is the position of a spouse, and $X_{d, j}$ is the position of the individual which the $i$ th hen wants to steal food, where $c \in\left\{1,2,3, \cdots, N_{r}\right\}, d \in\left\{1,2,3, \cdots, N_{h}\right\}$. The stealing and searching abilities of hens are related to their fitness values. The smaller the fitness value is, the stronger the foraging and stealing abilities are. These foraging behaviors are described by the following (13)-(15):

$$
\begin{gathered}
X_{i, j}^{\text {new }}=X_{i, j}+S_{1} \cdot \Re \text { and } \cdot\left(X_{c, j}-X_{i, j}\right)+S_{2} \cdot \Re \text { and } \cdot\left(X_{d, j}-X_{i, j}\right) \\
S_{1}=\exp \left(\frac{f_{i}-f_{c}}{\left|f_{i}\right|+\zeta}\right) \\
S_{2}=\exp \left(f_{d}-f_{i}\right)
\end{gathered}
$$

where $\mathfrak{R}$ and is a random number ranging within $[0,1]$.

\subsection{Chicken foraging}

Chicks follow their mothers to forage food. The smaller their fitness values, the easier for them to find food in the foraging process. The foraging behavior of the chicks is shown in (16):

$$
X_{i, j}^{\text {new }}=X_{i, j}+\varpi \cdot\left(X_{m, j}-X_{i, j}\right)
$$

where $X_{i, j}$ and $X_{i, j}^{\text {new }}$ are the original position and the updated position of each chick, respectively. For each chick, $i \in$ $\left\{N_{h}+1, N_{h}+2, \cdots, N_{m}\right\}$, and $j \in\{1,2,3, \cdots, D\} . X_{m, j}$ is the position of the mother hen corresponding to the $i$ th chick, where 
$m \in\left\{N_{r}+1, N_{r}+2, \cdots, N_{h}\right\} . \varpi$ is the following probability, which indicates the probability for each chick following its mother-hen to forage. Considering the differences between each chick, $\varpi$ is randomly generated between 0 and 2 .

\subsection{Algorithm framework}

The pseudo-code of CSO is shown in Algorithm 1.

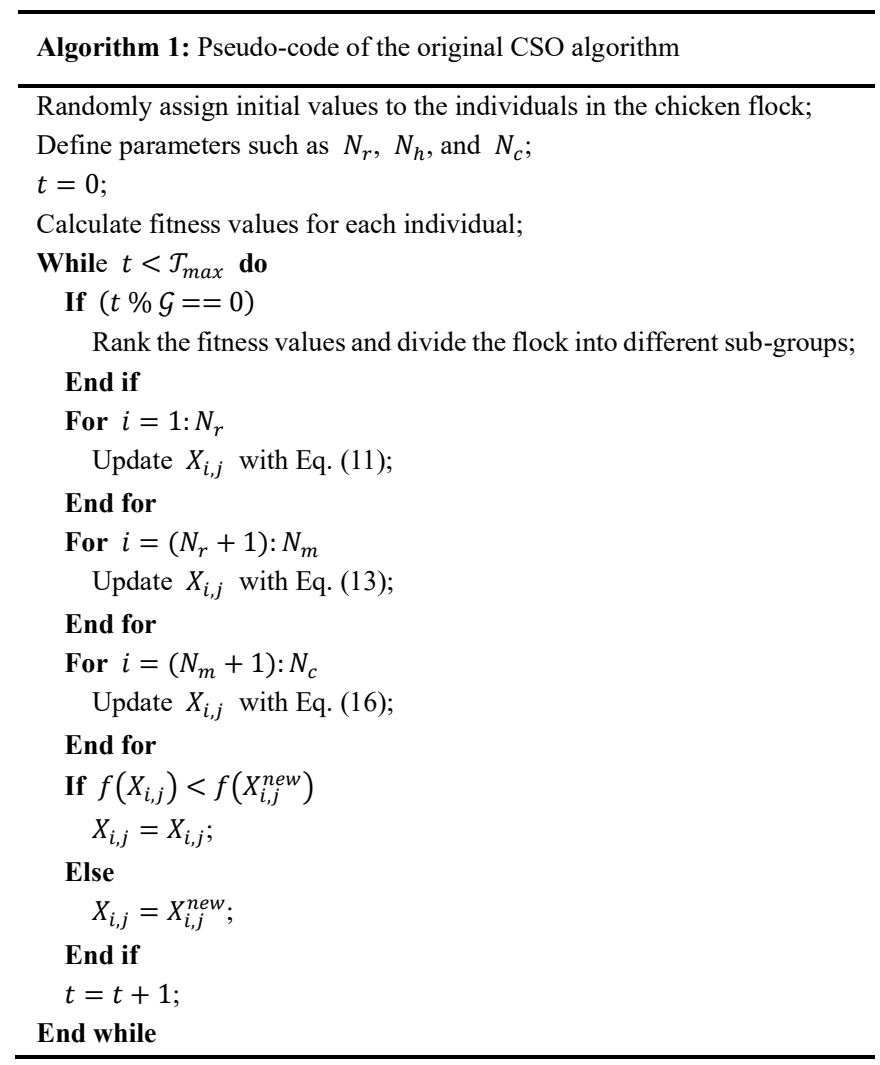

Here, $t$ is the current iteration number, and $\mathcal{T}_{\max }$ is the maximum iteration number.

\section{Proposed SCCSO algorithm}

\subsection{Motivation}

Although CSO performs excellently in many optimization problems, there still exist some shortcomings. First, the roosters in the rooster sub-flock only learn from a randomly-selected rooster, which causes the information of other roosters cannot be fully utilized. Second, the chicks neither learn from the rooster when foraging nor use the latest information in the chicken flock. Thus, they may fail to concentrate near food effectively and degenerate the convergence speed. Third, the ability of CSO to jump out of the local optimum is not strong enough, because hens and chicks also have a high probability of falling into local optimum when roosters fall into the local optimum. To address these problems, SCCSO is proposed with three effective strategies.

\subsection{Grouping principle}

In SCCSO, all individuals are rearranged according to the fitness values of all individuals in ascending order, and thus the identity of each individual can be determined. The sorted individuals are divided into three sub-flocks: rooster sub-flock, hen subflock, and chicken sub-flock. Without loss of generality, SCCSO is used to search the minimum. Therefore, individuals with smaller fitness values are appointed as roosters while with the larger fitness values are chicks, and the rest are hens. Like CSO, SCCSO also follows the same regulation that the smaller the fitness value of chickens, the stronger the ability to find and search for food. The number of individuals in each sub-flock is represented by $\mathbb{N}_{r}, \mathbb{N}_{h}$, and $\mathbb{N}_{c}$, respectively, and their ratio is definded as $r$ with value of 1:2:1 in this paper. The total number of individuals is $N$, and $N=\mathbb{N}_{r}+\mathbb{N}_{h}+\mathbb{N}_{c}$.

\subsection{Improved strategy}

\subsubsection{Information sharing strategy}

The information-sharing strategy is developed to update roosters that are individuals with smaller fitness values. Specifically, $X_{r_{1}}$ and $X_{r_{2}}$ are the positions of two roosters that are randomly selected from the rooster sub-flock; without loss of generality, $X_{r_{1}}$ and $X_{r_{2}}$ are defined as the positions of the rooster with better and worse fitness values, respectively. For each $i \in$ $\left\{1,2,3, \cdots, \mathbb{N}_{r}\right\}, X_{i}$ is the position of the updated rooster at each iteration. It is allowed to follow $X_{r_{1}}$ for foraging food, however, it will steal food from $X_{r_{2}}$. In this way, each updated rooster has access to share the information of other roosters, thus improving the exploitation ability. From the above analysis, the position of each rooster is updated by (17).

$$
X_{i}^{\text {new }}=X_{i}+\Re \text { andn } *\left[\left(X_{r_{1}}-X_{i}\right)-\left(X_{r_{2}}-X_{i}\right)\right]
$$


where $X_{i}^{\text {new }}$ is the updated position of the $i$ th rooster. $\Re a n d n$ is a standard normal distribution and its mean value and the standard deviation is set to 0 and 1 , respectively. If the updated position of a rooster is not good as the original position, the rooster will ignore the updated position and stays at the original position until the next update.

\subsubsection{Spiral movement strategy}

The spiral movement strategy is introduced to improve the foraging behaviors of the hens and chicks. First, for each hen, its position is defined as $X_{i}$, where $i \in\left\{\mathbb{N}_{r}+1, \mathbb{N}_{r}+2, \cdots, \mathbb{N}_{r}+\mathbb{N}_{h}\right\}$. The spouse of each hen is randomly selected from the rooster sub-flock whose position is defined as $X_{r}$, where $r \in\left\{1,2,3, \cdots, \mathbb{N}_{r}\right\}$. The updated position of each hen can be obtained by the distance between the hen and its spouse with the spiral movement strategy as follows:

$$
\left\{\begin{array}{l}
X_{i}^{\text {new }}=\vartheta_{1} * \Re a \text { and } n * D i s_{i, r} * \exp (b l) * \cos (2 \pi l)+X_{r} \\
\text { Disis }_{i, r}=X_{r}-X_{i}
\end{array}\right.
$$

where $D i s_{i, r}$ means the distance between each hen and its spouse. $e^{b l} * \cos (2 \pi l)$ denotes the spiral movement strategy, which can effectively enhance the exploration capability due to the spiral movement. Specifically, the spiral movement allows each hen to bypass the rooster and explore a wider space, instead of being limited to exploring the searching space between them. Here, $b$ is a constant for defining the shape of the spiral movement, and it is set to -2 in this paper. $l$ is a random number in the range $[-1,1]$, shown in (19) as follows. Particularly, $\vartheta_{1}$ is a variable parameter and here the value is set to 4 .

$$
\begin{aligned}
& l=(a-1) * \Re \text { and }+1 \\
& a=u *\left(1+\frac{F E S}{F E S_{\max }}\right)
\end{aligned}
$$

where $\Re$ and is a random number ranging between $[0,1] . u$ is set to -0.5 . FES is the current iteration number and FES $S_{\max }$ is the maximum iteration number.

Afterward, for each chick, its position is also defined as $X_{i}$, where $i \in\left\{\mathbb{N}_{r}+\mathbb{N}_{h}+1, \mathbb{N}_{r}+\mathbb{N}_{h}+1, \cdots, N\right\}$. At the early iteration stage, each chick follows a rooster for foraging food while at the later iteration stage, it helps a mother-hen to seek food and thus move towards the mother-hen. Therefore, a rooster and a mother-hen are first randomly selected from the rooster and hen sub-flock and their positions are respectively defined as $X_{r}$ and $X_{m}$, where $r \in\left\{1,2,3, \cdots, \mathbb{N}_{r}\right\}, m \in\left\{\mathbb{N}_{r}+1, \mathbb{N}_{r}+2, \cdots, \mathbb{N}_{r}+\right.$ $\left.\mathbb{N}_{h}\right\}$. The updated position of each chick can be obtained by the distance between each chick and its corresponding rooster with the same spiral movement strategy at the early iteration stage:

$$
\left\{\begin{array}{l}
X_{i}^{\text {new }}=\vartheta_{2} * \Re \text { and } n * D i s_{i, r} * \exp (b l) * \cos (2 \pi l)+X_{r} \\
\text { Dis }_{i, r}=X_{r}-X_{i}
\end{array}\right.
$$

where $D i s_{i, r}$ indicates the distance between the $i$ th chick and its corresponding rooster. $\vartheta_{2}$ is a variable parameter and set to 3 . The setting values of the other parameters are the same as those mentioned above.

Conversely, at the later iteration stage, the updated position of each chick can be represented by the distance between each chick and its corresponding mother-hen with the spiral movement strategy:

$$
\left\{\begin{array}{l}
X_{i}^{\text {new }}=\vartheta_{2} * \Re a \text { and } n * D i s_{i, m} * \exp (b l) * \cos (2 \pi l)+X_{m} \\
\text { Dis } s_{i, m}=X_{m}-X_{i}
\end{array}\right.
$$

where $D i s_{i, r}$ is the distance between the $i$ th chick and its corresponding mother-hen. In this strategy, both chicks and hens moved towards the roosters with a spiral trajectory at the early integration stage. The whole chicken flock can explore a wider searching range, and gather near the food quickly, thus improving the convergence speed. At the later iteration, chicks have almost gathered around the food. Chicks follow their mothers to search for food, which is beneficial to local exploitation.

\subsubsection{Self-adaptive-based chaotic disturbance mechanism}

The chaotic maps can create a chaotic sequence with better dynamic and statistics properties in a specific order (Ismail et al. 2019). Compared with random numbers created by imposing ordinary probability distributions, the use of chaotic sequences helps the individuals to perform searches at a higher speed (Coelho and Mariani 2008). By increasing the convergence speed and preventing sinking into the local optimum, the chaotic sequence can be used to improve the performance of the solution obtained by the individuals (Lu et al. 2014).

Due to such a fact, a self-adaptive-based chaotic disturbance mechanism is developed for guiding the movement of the individual with the worst fitness value to move towards the leader rooster at each iteration, which can improve the overall quality of the chicken flock. Assume that the position of the individual with the worst fitness value is defined as $X_{w}$, and its updated position is defined as $X_{w}^{\text {new }}$. In the early iteration stage, $X_{w}^{\text {new }}$ is generated by imposing a certain disturbance on the leader rooster whose position is defined as $X_{b}$. And in the later iteration stage, $X_{b}$ may be very close to the food, so it is necessary to retain more information about $X_{b}$ for $X_{w}^{\text {new }}$. With the self-adaptive-based chaotic disturbance mechanism, the updating method of $X_{w}$ is given as follows:

$$
\begin{aligned}
X_{w}^{\text {new }}=\left\{\begin{aligned}
X_{b}, \text { if } \Re \text { and }_{2} \geq 1-\frac{F E S}{F E S_{\max }} \\
X_{b}+\Re \text { and }_{1} *\left(2 C_{k}-1\right), \text { otherwise }
\end{aligned}\right. \\
C_{k+1}=4 * C_{k} *\left(1-C_{k}\right)
\end{aligned}
$$

where $\Re a n d_{1}$ and $\Re a n d_{2}$ are different random numbers generating within $[0,1] . k$ is the iteration number, $C_{k}$ is the value at $k$ th chaotic iteration, and the initial value of the chaotic sequence is randomly generated within $[0,1]$. The result obtained by $1-\frac{F E S}{F E S_{\max }}$ is a self-adaptive changed value. According to the comparison between the self-adaptive changed value and $\mathfrak{R a n d}_{2}$, there is no obvious boundary between pre-iteration and post-iteration so that the natural transition is realized. 
Based on the above descriptions, the flow chart of SCCSO is shown in Fig. $4^{3}$, and the pseudo-code of SCCSO is summarized 310 in Algorithm 2.

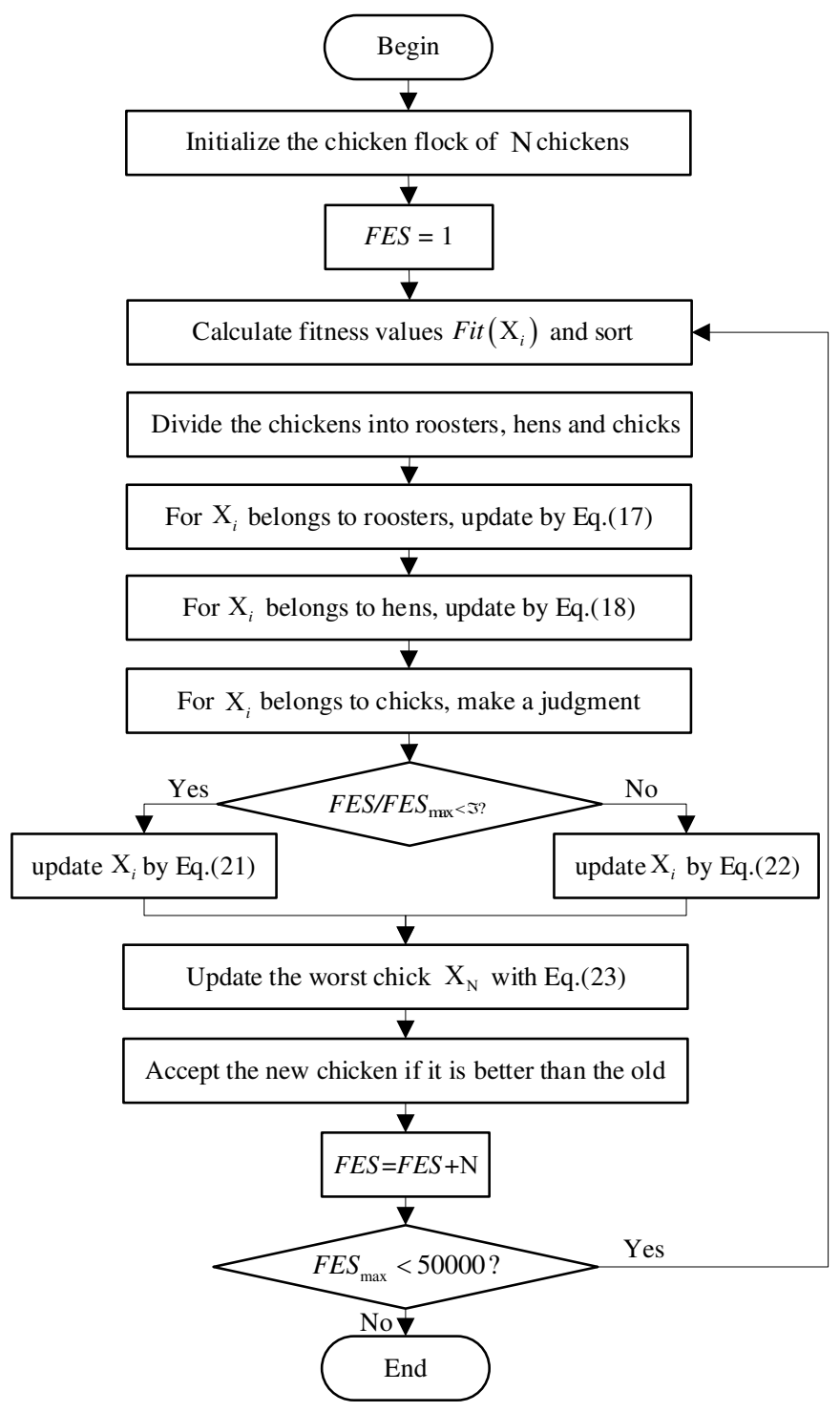

Fig. 4 Flow chart of SCCSO

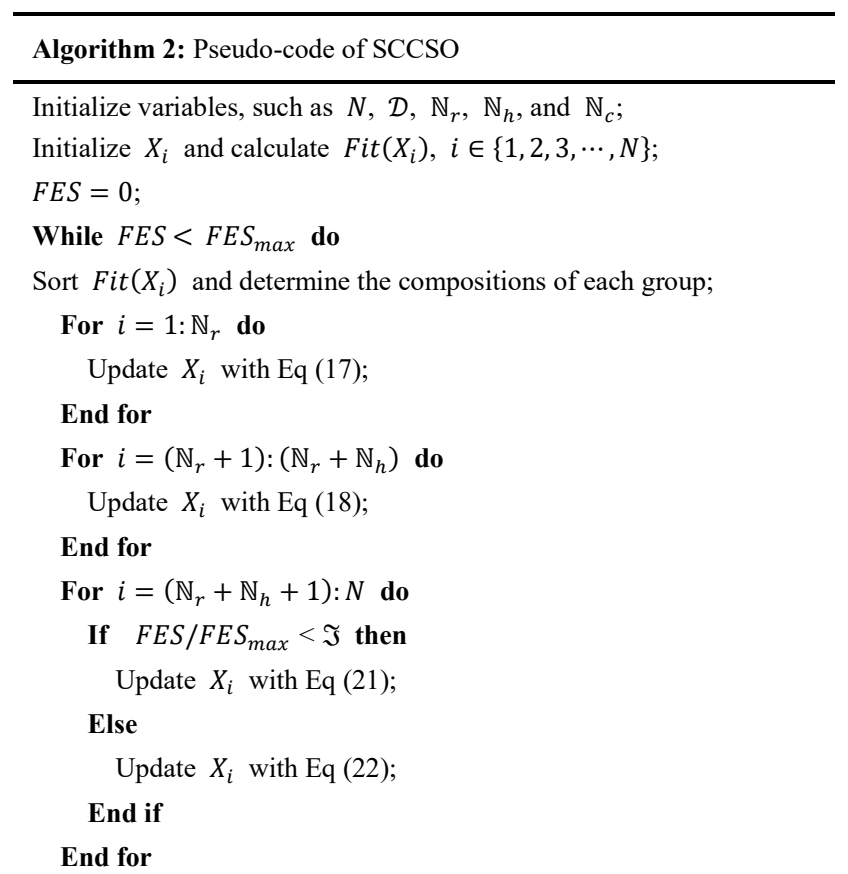


Here, $\mathcal{D}$ is the dimension of the searching range. Fit is the fitness function and Fit $\left(X_{i}\right)$ expresses the fitness value of each individual in the chicken flock. $\mathfrak{J}$ is the constant controlling the time that the chicks follow the roosters foraging, here it is set to 0 .

\subsection{Time computational complexity of SCCSO}

Following is the time computational complexities of SCCSO:

(1) Initialization requires $O(N \times \mathcal{D})$ time, where $N$ represents the size of the chicken population, and $\mathcal{D}$ is the dimension of the searching range, $N \gg \mathcal{D}$.

(2) Fitness calculation requires $O(N \times \mathcal{D})$ time.

(3) Fitness sorting requires $O(N \times \log N)$ time.

(4) Position updating requires $O(N \times \mathcal{D})$ time.

For $N \gg \mathcal{D}$, so $O(N \times \log N) \gg O(N \times \mathcal{D})$. In conclusion, the total time complexity is $\mathrm{O}(N \times \log N)$ time per generation, the same as CSO's. Therefore, the total time complexity of SCCSO for maximum iteration number is $O\left(N \times \log N \times F E S_{\max }\right)$, where $F E S_{\max }$ is the maximum iteration number.

\section{Experimental results analysis and discussion}

To verify the effectiveness of SCCSO, it is applied to the parameters identification of different PV models, such as single diode, double diode, and PV module models. The current and voltage data of various solar cells and modules originates from reference (Easwarakhanthan et al. 1986), which has been vastly applied in various techniques of PV models' parameters identification (Chen et al. 2016; Gong et al.; Niu et al. 2014; Oliv et al. 2017; Yu et al. 2017b). In reference, the commercial R.T.C. French silicon solar cells, $57 \mathrm{~mm}$ in diameter, operates at $33^{\circ} \mathrm{C}$ at an irradiance of $1000 \mathrm{~W} / \mathrm{m}^{2}$. A solar module consisting of 36 polysilicon cells in series, called photowATt-PWP201, operates at $45^{\circ} \mathrm{C}$ at an irradiance of $1000 \mathrm{~W} / \mathrm{m}^{2}$. Table 1 shows the rational upper and lower limitations of different PV model parameters.

Table 1 Parameters ranges of three PV models

\begin{tabular}{|c|c|c|c|c|}
\hline \multirow{2}{*}{ Parameter } & \multicolumn{2}{|c|}{ Single/Double diode } & \multicolumn{2}{|c|}{ PV module } \\
\hline & Lower bound & Upper bound & Lower bound & Upper bound \\
\hline$I_{P H}(\mathrm{~A})$ & 0 & 1 & 0 & 2 \\
\hline$I_{S D}, I_{S D 1}, I_{S D 2}(\mu \mathrm{A})$ & 0 & 1 & 0 & 50 \\
\hline$R_{L}(\Omega)$ & 0 & 0.5 & 0 & 2 \\
\hline $\boldsymbol{R}_{S H}(\Omega)$ & 0 & 100 & 0 & 2000 \\
\hline$n, n_{1}, n_{2}$ & 1 & 2 & 1 & 50 \\
\hline
\end{tabular}

To demonstrate the competitive performance of SCCSO, it is compared with seven other advanced algorithms which perform excellently in the parameter identification of PV models. They are chicken swarm optimization (CSO) (Meng et al. 2014), artificial bee colony algorithm (Karaboga and Ozturk 2011), moth flame optimization algorithm (MFO) (Mirjalili 2015), improved JAYA optimization algorithm (IJAYA) (Yu et al. 2017b), performance-guided JAYA algorithm (PGJAYA) (Yu et al. 2019), improved brainstorming optimization algorithm (IBSO) (Yan et al. 2019) and improved moth-flame optimization (IMFO) (Sheng et al. 2019). According to the recommendations in the corresponding references, Table 2 shows the parameter configuration of the algorithms involved. For fairness, the maximum evaluation time and run number of each algorithm in each experiment are set to 50000 and 30 , respectively.

Table 2 Parameter configuration of different algorithms

\begin{tabular}{cc}
\hline Algorithm & Parameter configuration \\
\hline SCCSO & $N=100, R N=30, M N=40, C N=30, t=0.25$ \\
CSO (Meng et al. 2014) & $N=100, R N=15, H N=70, M N=50, C N=15, G=20, F L \in[0.4,0.9]$ \\
ABC (Karaboga and Ozturk 2011) & $N=50, D=5$, limit $=N * D$ \\
MFO (Mirjalili 2015) & $N=50$ \\
IMFO (Sheng et al. 2019) & $N=20$ \\
IJAYA (Yu et al. 2017b) & $N=20$ \\
PGJAYA (Yu et al. 2019) & $N=50, M=5, r_{1}=$ rand, $p_{r 1}=0.8$ \\
IBSO (Yan et al. 2019) & $N=0.4$ \\
\hline
\end{tabular}

In the following sections, comparisons are conducted first on the performance of each algorithm about accuracy, robustness, and convergence speed by analyzing experimental results and convergence curves. Next, comparisons are made for the best RMSE values gained by the above-mentioned algorithms for 30 runs. Addtionally, analysis are performed on the sensitivity of crucial parameters. Then, authentication and disscussion are made about the effectiveness of the strategies proposed in SCCSO. To show the statistical results clearly, the overall best and second-best results of RMSE are underlined in bold gray and bold, respectively. 
In this section, we evaluated the performance of all advanced algorithms including accuracy, robustness, and convergence speed by analyzing experimental data and convergence curve. Experimental data are shown in Table 3, where the minimum (Min) and average (Mean) value of RMSE reflects the accuracy and average accuracy of algorithms, respectively. The standard deviation of RMSE (SD) is related to the reliability of algorithms. Addtionally, the Wilcoxon signed-rank test with a significant level of $5 \%$ (Alcalá-Fdez et al. 2008) is introduced to estimate the difference between SCCSO and other algorithms. "+" and " $\approx$ " represent the performance of SCCSO is significantly better than or similar to that of other algorithms, respectively.

Table 3 Experimental results of different algorithms for three models

\begin{tabular}{|c|c|c|c|c|c|c|}
\hline \multirow{2}{*}{ Model } & \multirow{2}{*}{ Algorithm } & \multicolumn{4}{|c|}{ RMSE } & \multirow{2}{*}{$\begin{array}{l}\text { Wilcoxon signed- } \\
\text { rank test }\end{array}$} \\
\hline & & Min & Mean & Max & SD & \\
\hline \multirow{8}{*}{$\begin{array}{l}\text { Single diode } \\
\text { model }\end{array}$} & SCCSO & $9.8602 \mathrm{E}-04$ & $9.8602 \mathrm{E}-04$ & $9.8602 \mathrm{E}-04$ & 2.3572E-17 & \\
\hline & $\mathrm{CSO}$ & $1.0004 \mathrm{E}-03$ & $1.4457 \mathrm{E}-03$ & $2.1624 \mathrm{E}-03$ & $3.7225 \mathrm{E}-04$ & + \\
\hline & $\mathrm{ABC}$ & $9.9278 \mathrm{E}-04$ & $1.4075 \mathrm{E}-03$ & $1.1394 \mathrm{E}-03$ & $1.0587 \mathrm{E}-04$ & + \\
\hline & MFO & $9.9272 \mathrm{E}-04$ & $2.0594 \mathrm{E}-03$ & 4.7694E-03 & $6.5366 \mathrm{E}-04$ & + \\
\hline & IMFO & $9.8603 \mathrm{E}-04$ & $9.8999 \mathrm{E}-04$ & $1.0212 \mathrm{E}-03$ & $6.8178 \mathrm{E}-06$ & + \\
\hline & IJAYA & $9.8608 \mathrm{E}-04$ & $9.9132 \mathrm{E}-04$ & $1.0419 \mathrm{E}-03$ & $1.0010 \mathrm{E}-05$ & + \\
\hline & PGJAYA & $9.8602 E-04$ & $9.8602 \mathrm{E}-04$ & $9.8603 E-04$ & $8.1518 \mathrm{E}-10$ & + \\
\hline & IBSO & $9.8602 E-04$ & $9.8605 E-04$ & $9.8627 \mathrm{E}-04$ & $6.5208 \mathrm{E}-08$ & + \\
\hline \multirow{8}{*}{$\begin{array}{l}\text { Double diode } \\
\text { model }\end{array}$} & SCCSO & $9.8248 \mathrm{E}-04$ & $9.8366 \mathrm{E}-04$ & $9.8609 \mathrm{E}-04$ & $1.4171 \mathrm{E}-06$ & \\
\hline & $\mathrm{CSO}$ & $9.9273 \mathrm{E}-04$ & $1.9532 \mathrm{E}-03$ & $2.9254 \mathrm{E}-03$ & $5.2375 \mathrm{E}-04$ & + \\
\hline & $\mathrm{ABC}$ & $9.9505 \mathrm{E}-04$ & $1.2079 \mathrm{E}-03$ & $1.0660 \mathrm{E}-03$ & 5.9047E-05 & + \\
\hline & MFO & $9.8287 E-04$ & $3.1610 \mathrm{E}-03$ & $3.3398 \mathrm{E}-02$ & $5.7468 \mathrm{E}-03$ & + \\
\hline & IMFO & $9.8298 \mathrm{E}-04$ & $1.0635 \mathrm{E}-03$ & $1.8118 \mathrm{E}-03$ & $1.6481 \mathrm{E}-04$ & + \\
\hline & IJAYA & $9.8388 \mathrm{E}-04$ & 1.0192E-03 & $1.4391 \mathrm{E}-03$ & $9.9613 \mathrm{E}-05$ & + \\
\hline & PGJAYA & $9.8294 \mathrm{E}-04$ & $9.8680 \mathrm{E}-04$ & 1.0042E-03 & $3.8186 \mathrm{E}-06$ & + \\
\hline & IBSO & $9.8554 \mathrm{E}-04$ & $1.0259 \mathrm{E}-03$ & $1.3097 \mathrm{E}-03$ & $7.4098 \mathrm{E}-05$ & + \\
\hline \multirow{8}{*}{$\begin{array}{l}\text { PV module } \\
\text { modle }\end{array}$} & SCCSO & $2.4251 \mathrm{E}-03$ & $2.4251 \mathrm{E}-03$ & $2.4251 \mathrm{E}-03$ & $1.5950 \mathrm{E}-17$ & \\
\hline & $\mathrm{CSO}$ & $2.4333 \mathrm{E}-03$ & $2.5366 \mathrm{E}-03$ & $2.7329 \mathrm{E}-03$ & $6.9708 \mathrm{E}-05$ & + \\
\hline & $\mathrm{ABC}$ & $2.4444 \mathrm{E}-03$ & 2.6307E-03 & $2.5661 \mathrm{E}-03$ & $5.4815 \mathrm{E}-05$ & + \\
\hline & MFO & $2.4982 \mathrm{E}-03$ & $2.6051 \mathrm{E}-03$ & $2.7428 \mathrm{E}-03$ & $3.4529 \mathrm{E}-05$ & + \\
\hline & IMFO & $2.4256 \mathrm{E}-03$ & $2.4510 \mathrm{E}-03$ & $2.7083 \mathrm{E}-03$ & $6.8809 \mathrm{E}-05$ & + \\
\hline & IJAYA & $2.4252 \mathrm{E}-03$ & $2.4309 \mathrm{E}-03$ & $2.4639 \mathrm{E}-03$ & 7.9201E-06 & + \\
\hline & PGJAYA & $2.4251 \mathrm{E}-03$ & 2.4253E-03 & $2.4289 \mathrm{E}-03$ & $8.3628 \mathrm{E}-07$ & + \\
\hline & IBSO & $2.4251 \mathrm{E}-03$ & $2.4251 \mathrm{E}-03$ & $2.4252 \mathrm{E}-03$ & 2.0783E-08 & + \\
\hline
\end{tabular}

In Table 3, the results obtained by SCCSO on the three models are superior to the other seven advanced algorithms in accuracy and reliability. More specifically, in the single diode model, besides SCCSO, PGJAYA and IBSO also obtain the best Min, and IMFO gains the second best. Although PGJAYA also gets the best Mean, its reliability is inferior to SCCSO. In the double diode model, SCCSO also performs best as it gets the best in all aspects except for a small gap between SD and the best SD. In the PV module model, SCCSO also achieves best results in all aspects. Besides, IBSO and PGJAYA obtain the best Min, and IBSO ranks second in accuracy and reliability. Obviously, the Wilcoxon signed-rank test results show the superior performance of SCCSO on all mentioned PV models to other competitive algorithms.

Furthermore, boxplots are applied to visually show the distribution of results obtained by every algorithm in 30 independent runs on three models, as shown in Fig. $5^{4}$. Note that the symbol "+" represents the disordered values. Comparing the span of solution distributions and we can see that SCCSO performs better in accuracy and robustness than other advanced algorithms.

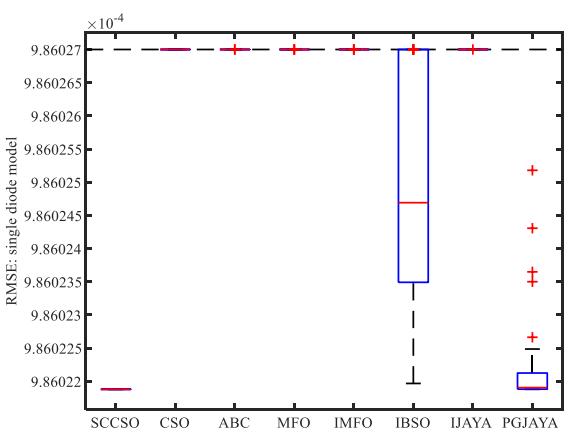

(a)

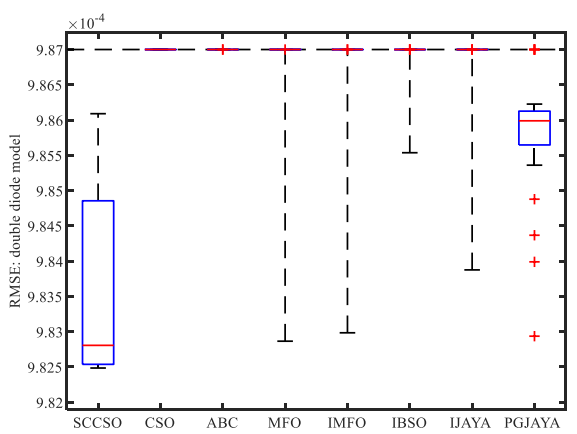

(b)

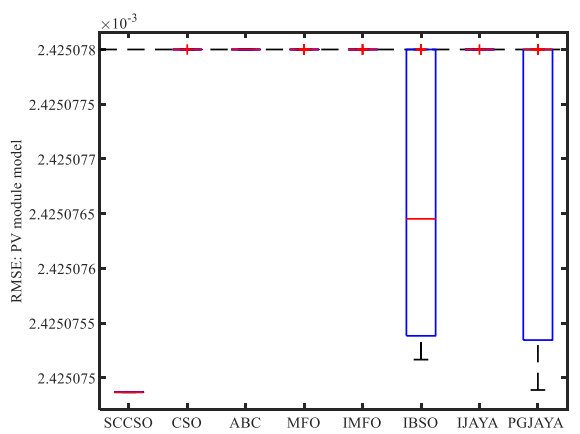

(c)

Fig. 5 Best RMSE boxplot in 30 runs of different algorithms for: (a) single diode model, (b) double diode model, (c) PV module model

\footnotetext{
${ }^{4}$ The figure was drawn with MATLAB.
} 
To investigate the computational efficiency of SCCSO, Fig. $6^{4}$ shows the average computational time of all algorithms on three models in 30 runs. For each algorithm, the CPU time are obtained on a PC Intel Core 7 Duo $1.80 \mathrm{GHz}$ with a $8 \mathrm{~GB}$ RAM that runs on Windows 10 with MATLAB R2018a implementation. From Fig. 6, the proposed algorithm and PGJAYA consumes the lowest computational overhead among all algorithms. This indicates that SCCSO can obtain superior results under the condition of limited computational overhead.

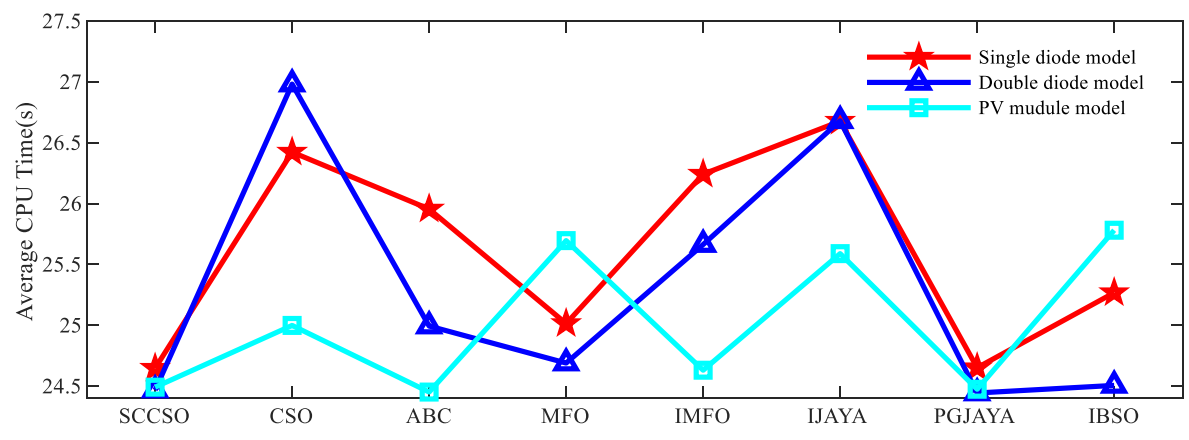

Fig. 6 The average CPU time of different algorithms for three models

According to the average RMSE values obtained by each algorithm, the convergence curves are drawn and shown in Fig. $7^{4}$. By amplifying the curves, it is obvious that SCCSO performs excellently in convergence speed as it gets a fast speed and obtains the best convergence values in the end among all algorithms.

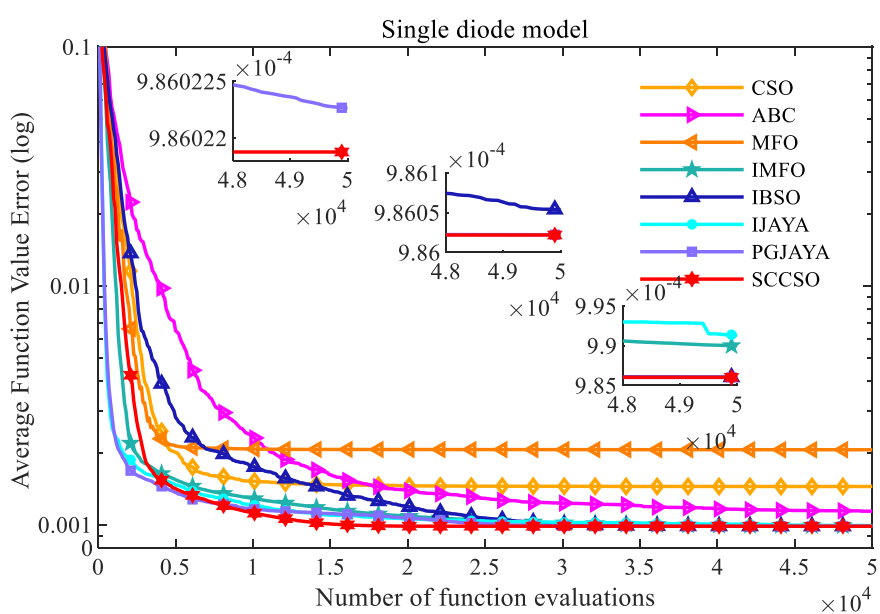

(a)

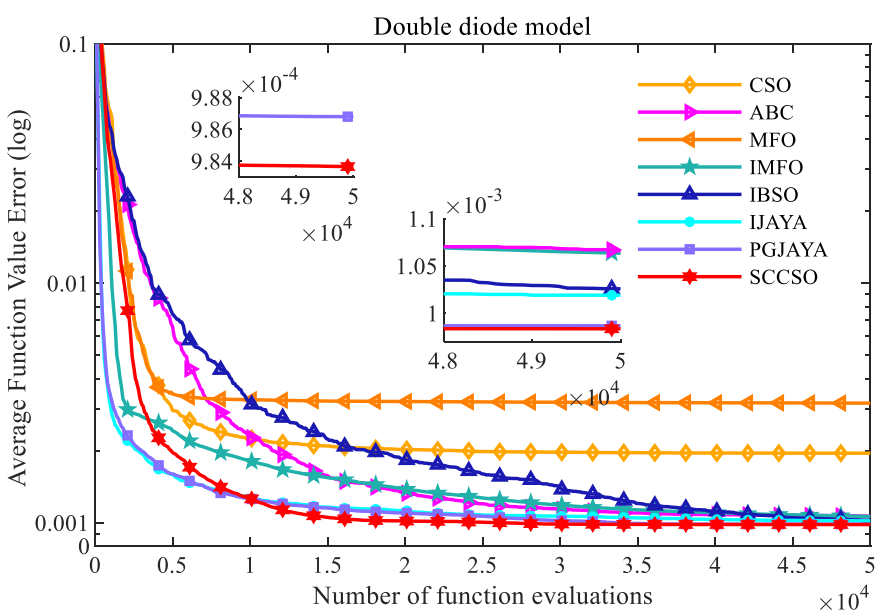

(b)

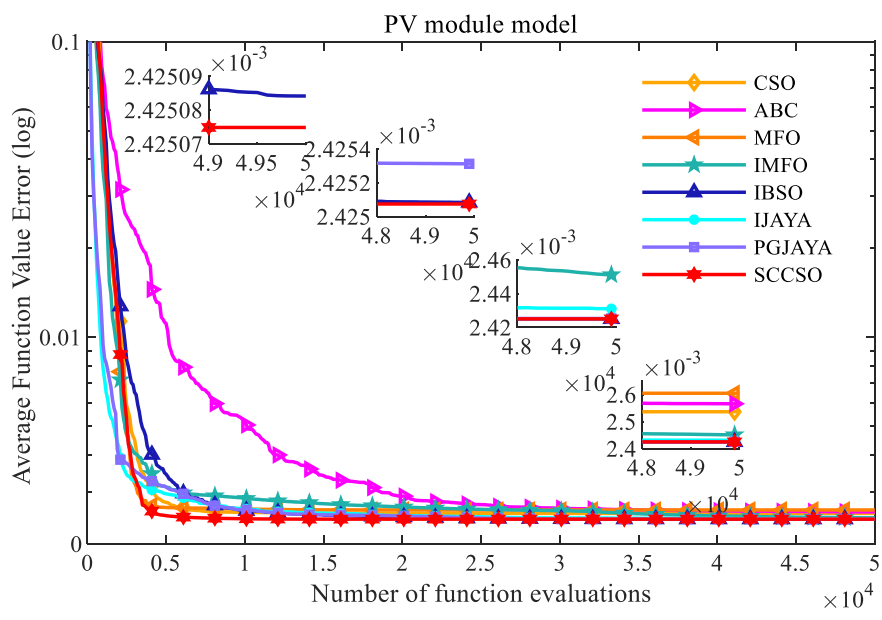

(c)

Fig. 7 Convergence curves of SCCSO and compared algorithms for: (a) single diode model, (b) double diode model, (c) PV module model

To summarize, the above analysis and comparisons of experimental results show that SCCSO performs better in robustness, accuracy, and convergence speed compared with the other competetive algorithms.

\subsection{Detailed experimental results analysis}

In this section, the best RMSE values and the related parameters identified by different algorithms in 30 independent runs are analyzed detailedly. 


\subsubsection{Single diode model}

Table 4 presents the analyzed experimental results on the single diode model, including the adjusted five parameters and the related RMSE values. We can observe that SCCSO, PGJAYA, and IBSO acquire the best RMSE value (9.8602E-04), while IMFO achieves the second-best value (9.8603E-04), followed by IJAYA, MFO, ABC, and CSO. Although the difference of RMSE value corresponding to each algorithm is not much different, a slight difference can improve the accuracy of adjusted parameters significantly for the objective function. Besides, Table 5 shows the individual absolute errors (IAE) between the experimental data and simulated data (Easwarakhanthan et al. 1986). All the IAE values of current are no more than 0.02152687 while those of power are less than 0.00873078 , which shows the accuracy of the adjusted five parameters.

Addionally, the best-adjusted parameters obtained by SCCSO are applied to constract the I-V and P-V curves in Fig. $8^{4}$. Clearly, the simulated data acquired by SCCSO (blue five-pointed stars) are extremely unanimous with the experimental data (purple lines) within the whole voltage range.

Table 4 Comparisons of experimental results on single diode model

\begin{tabular}{|c|c|c|c|c|c|c|}
\hline Algorithm & $I_{P H}(\mathrm{~A})$ & $I_{S D}(\mu \mathrm{A})$ & $R_{L}(\Omega)$ & $R_{S H}(\Omega)$ & $n$ & $R M S E$ \\
\hline SCCSO & 0.7608 & 0.32302 & 0.0364 & 53.7185 & 1.4812 & $9.8602 \mathrm{E}-04$ \\
\hline $\mathrm{CSO}$ & 0.7607 & 0.35248 & 0.0360 & 56.7696 & 1.4900 & $1.0004 \mathrm{E}-03$ \\
\hline $\mathrm{ABC}$ & 0.7608 & 0.30534 & 0.0366 & 52.3455 & 1.4755 & $9.9278 \mathrm{E}-04$ \\
\hline MFO & 0.7607 & 0.34312 & 0.0361 & 55.4217 & 1.4873 & $9.9272 \mathrm{E}-04$ \\
\hline IMFO & 0.7608 & 0.32225 & 0.0364 & 53.6451 & 1.4809 & $9.8603 E-04$ \\
\hline IJAYA & 0.7608 & 0.32312 & 0.0364 & 53.6988 & 1.4812 & $9.8608 \mathrm{E}-04$ \\
\hline PGJAYA & 0.7608 & 0.32302 & 0.0364 & 53.7189 & 1.4812 & $9.8602 \mathrm{E}-04$ \\
\hline IBSO & 0.7608 & 0.32299 & 0.0364 & 53.7230 & 1.4812 & $9.8602 \mathrm{E}-04$ \\
\hline
\end{tabular}

Table 5 IAE of SCCSO on single diode model

\begin{tabular}{|c|c|c|c|c|c|c|c|}
\hline \multirow{2}{*}{ Item } & \multicolumn{2}{|c|}{ Measured data } & \multirow{2}{*}{$\frac{\text { Calculated data }}{P(W)}$} & \multicolumn{2}{|c|}{ Simulated current data } & \multicolumn{2}{|c|}{ Simulated power data } \\
\hline & $U(V)$ & $I(A)$ & & $I_{\text {sim }}(A)$ & $I A E_{I}$ & $P_{\text {sim }}(W)$ & $I A E_{P}$ \\
\hline 1 & -0.2057 & 0.7640 & -0.1572 & 0.76408770 & 0.00008770 & -0.15717284 & 0.00001804 \\
\hline 2 & -0.1291 & 0.7620 & -0.0984 & 0.76266309 & 0.00066309 & -0.09845980 & 0.00008560 \\
\hline 3 & -0.0588 & 0.7605 & -0.0447 & 0.76135531 & 0.00085531 & -0.04476769 & 0.00005029 \\
\hline 4 & 0.0057 & 0.7605 & 0.0043 & 0.76015399 & 0.00034601 & 0.00433288 & 0.00000197 \\
\hline 5 & 0.0646 & 0.7600 & 0.0491 & 0.75905521 & 0.00094479 & 0.04903497 & 0.00006103 \\
\hline 6 & 0.1185 & 0.7590 & 0.0899 & 0.75804235 & 0.00095765 & 0.08982802 & 0.00011348 \\
\hline 7 & 0.1678 & 0.7570 & 0.1270 & 0.75709165 & 0.00009165 & 0.12703998 & 0.00001538 \\
\hline 8 & 0.2132 & 0.7570 & 0.1614 & 0.75614136 & 0.00085864 & 0.16120934 & 0.00018306 \\
\hline 9 & 0.2545 & 0.7555 & 0.1923 & 0.75508687 & 0.00041313 & 0.19216961 & 0.00010514 \\
\hline 10 & 0.2924 & 0.7540 & 0.2205 & 0.75366388 & 0.00033612 & 0.22037132 & 0.00009828 \\
\hline 11 & 0.3269 & 0.7505 & 0.2453 & 0.75139097 & 0.00089097 & 0.24562971 & 0.00029126 \\
\hline 12 & 0.3585 & 0.7465 & 0.2676 & 0.74735385 & 0.00085385 & 0.26792636 & 0.00030611 \\
\hline 13 & 0.3873 & 0.7385 & 0.2860 & 0.74011722 & 0.00161722 & 0.28664740 & 0.00062635 \\
\hline 14 & 0.4137 & 0.7280 & 0.3012 & 0.72738222 & 0.00061778 & 0.30091803 & 0.00025557 \\
\hline 15 & 0.4373 & 0.7065 & 0.3090 & 0.70697265 & 0.00047265 & 0.30915914 & 0.00020669 \\
\hline 16 & 0.4590 & 0.6755 & 0.3101 & 0.67528015 & 0.00021985 & 0.30995359 & 0.00010091 \\
\hline 17 & 0.4784 & 0.6320 & 0.3023 & 0.63075827 & 0.00124173 & 0.30175476 & 0.00059404 \\
\hline 18 & 0.4960 & 0.5730 & 0.2842 & 0.57192836 & 0.00107164 & 0.28367647 & 0.00053153 \\
\hline 19 & 0.5119 & 0.4990 & 0.2554 & 0.49960702 & 0.00060702 & 0.25574883 & 0.00031073 \\
\hline 20 & 0.5265 & 0.4130 & 0.2174 & 0.41364879 & 0.00064879 & 0.21778609 & 0.00034159 \\
\hline 21 & 0.5398 & 0.3165 & 0.1708 & 0.31751011 & 0.00101011 & 0.17139196 & 0.00054526 \\
\hline 22 & 0.5521 & 0.2120 & 0.1170 & 0.21215494 & 0.00015494 & 0.11713074 & 0.00008554 \\
\hline 23 & 0.5633 & 0.1035 & 0.0583 & 0.10225131 & 0.00124869 & 0.05759816 & 0.00070339 \\
\hline 24 & 0.5736 & -0.0100 & -0.0057 & -0.00871754 & 0.00128246 & -0.00500038 & 0.00073562 \\
\hline 25 & 0.5833 & -0.1230 & -0.0717 & -0.12550741 & 0.00250741 & -0.07320847 & 0.00146257 \\
\hline 26 & 0.5900 & -0.2100 & -0.1239 & -0.20847233 & 0.00152767 & -0.12299867 & 0.00090133 \\
\hline Sum of IAE & & & & & 0.02152687 & & 0.00873078 \\
\hline
\end{tabular}

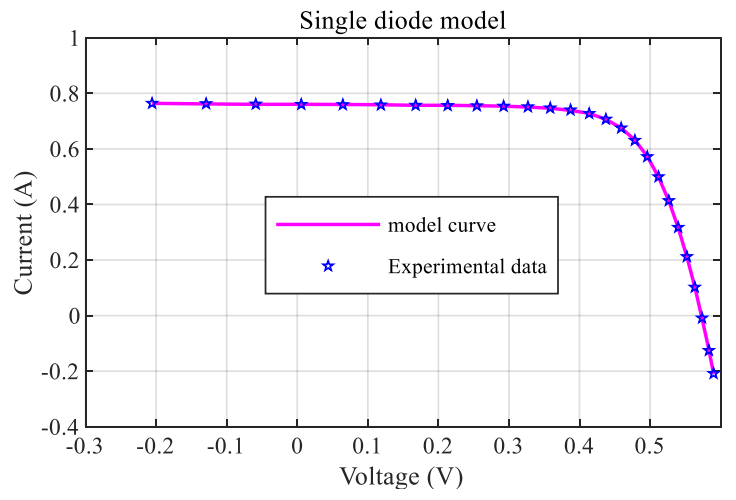

(a)

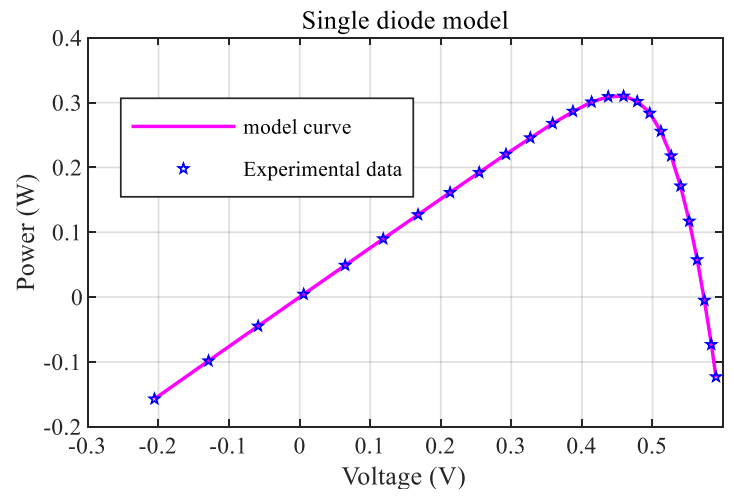

(b)

Fig. 8 Comparisons between experimental data and simulated data obtained by SCCSO on single diode model: (a) I-V characteristic, (b) P-V characteristic 


\subsubsection{Double diode model}

Table 6 lists the compared results of the adjusted seven parameters as well as the related RMSE values. Among all algorithms, SCCSO obtains the best RMSE value (9.8248E-04) while MFO gains the second-best value (9.8287E-04), followed by PGJAYA, IMFO, IJAYA, IBSO, CSO, and ABC. Table 7 presents the IAE values of current and power, which are less than 0.02127523 and 0.00877664 , respectively. Thus, we can conclude that SCCSO can achieve high accuracy parameters.

From the I-V and P-V curves presented in Fig. $9^{4}$, the experimental and simulated data almost coincide with each other within the whole voltage range.

Table 6 Comparisons of experimental results on double diode model

\begin{tabular}{|c|c|c|c|c|c|c|c|c|}
\hline Algorithm & $I_{P H}(\mathrm{~A})$ & $I_{S D 1}(\mu \mathrm{A})$ & $R_{L}(\Omega)$ & $R_{S H}(\Omega)$ & $n_{1}$ & $I_{S D 2}(\mu \mathrm{A})$ & $n_{2}$ & $R M S E$ \\
\hline SCCSO & 0.7608 & 0.22597 & 0.0367 & 55.4854 & 1.4510 & 0.74935 & 2 & 9.8248E-04 \\
\hline $\mathrm{CSO}$ & 0.7608 & 0.03814 & 0.0362 & 54.6261 & 1.7628 & 0.32712 & 1.4836 & $9.9273 \mathrm{E}-04$ \\
\hline $\mathrm{ABC}$ & 0.7606 & 0.25109 & 0.0368 & 54.8424 & 1.4590 & 0.40557 & 1.9955 & $9.9505 \mathrm{E}-04$ \\
\hline MFO & 0.7608 & 0.25300 & 0.0366 & 55.1029 & 1.4606 & 0.53624 & 2 & 9.8287E-04 \\
\hline IMFO & 0.7608 & 0.23074 & 0.0367 & 55.2518 & 1.4531 & 0.60012 & 1.9574 & $9.8298 \mathrm{E}-04$ \\
\hline IJAYA & 0.7608 & 0.62044 & 0.0368 & 55.1757 & 1.9167 & 0.21129 & 1.4463 & $9.8388 \mathrm{E}-04$ \\
\hline PGJAYA & 0.7608 & 0.25443 & 0.0366 & 54.8098 & 1.4609 & 0.50389 & 2 & $9.8294 \mathrm{E}-04$ \\
\hline IBSO & 0.7608 & 0.30919 & 0.0364 & 54.0020 & 1.4777 & 0.08964 & 1.9324 & $9.8554 \mathrm{E}-04$ \\
\hline
\end{tabular}

Table 7 IAE of SCCSO on double diode model

\begin{tabular}{|c|c|c|c|c|c|c|c|}
\hline \multirow{2}{*}{ Item } & \multicolumn{2}{|c|}{ Measured data } & \multirow{2}{*}{$\frac{\text { Calculated data }}{P(W)}$} & \multicolumn{2}{|c|}{ Simulated current data } & \multicolumn{2}{|c|}{ Simulated power data } \\
\hline & $U(V)$ & $I(A)$ & & $I_{\text {sim }}(A)$ & $I A E_{I}$ & $P_{\text {sim }}(W)$ & $I A E_{P}$ \\
\hline 1 & -0.2057 & 0.7640 & -0.1572 & 0.76398341 & 0.00001659 & -0.15715139 & 0.00000341 \\
\hline 2 & -0.1291 & 0.7620 & -0.0984 & 0.76260410 & 0.00060410 & -0.09845219 & 0.00007799 \\
\hline 3 & -0.0588 & 0.7605 & -0.0447 & 0.76133770 & 0.00083770 & -0.04476666 & 0.00004926 \\
\hline 4 & 0.0057 & 0.7605 & 0.0043 & 0.76017379 & 0.00032621 & 0.00433299 & 0.00000186 \\
\hline 5 & 0.0646 & 0.7600 & 0.0491 & 0.75910768 & 0.00089232 & 0.04903836 & 0.00005764 \\
\hline 6 & 0.1185 & 0.7590 & 0.0899 & 0.75812142 & 0.00087858 & 0.08983739 & 0.00010411 \\
\hline 7 & 0.1678 & 0.7570 & 0.1270 & 0.75718861 & 0.00018861 & 0.12705625 & 0.00003165 \\
\hline 8 & 0.2132 & 0.7570 & 0.1614 & 0.75624361 & 0.00075639 & 0.16123114 & 0.00016126 \\
\hline 9 & 0.2545 & 0.7555 & 0.1923 & 0.75517730 & 0.00032270 & 0.19219262 & 0.00008213 \\
\hline 10 & 0.2924 & 0.7540 & 0.2205 & 0.75372235 & 0.00027765 & 0.22038842 & 0.00008118 \\
\hline 11 & 0.3269 & 0.7505 & 0.2453 & 0.75139913 & 0.00089913 & 0.24563238 & 0.00029393 \\
\hline 12 & 0.3585 & 0.7465 & 0.2676 & 0.74730144 & 0.00080144 & 0.26790757 & 0.00028732 \\
\hline 13 & 0.3873 & 0.7385 & 0.2860 & 0.74001066 & 0.00151066 & 0.28660613 & 0.00058508 \\
\hline 14 & 0.4137 & 0.7280 & 0.3012 & 0.72724695 & 0.00075305 & 0.30086206 & 0.00031154 \\
\hline 15 & 0.4373 & 0.7065 & 0.3090 & 0.70685030 & 0.00035030 & 0.30910564 & 0.00015319 \\
\hline 16 & 0.4590 & 0.6755 & 0.3101 & 0.67521054 & 0.00028946 & 0.30992164 & 0.00013286 \\
\hline 17 & 0.4784 & 0.6320 & 0.3023 & 0.63076076 & 0.00123924 & 0.30175595 & 0.00059285 \\
\hline 18 & 0.4960 & 0.5730 & 0.2842 & 0.57199473 & 0.00100527 & 0.28370939 & 0.00049861 \\
\hline 19 & 0.5119 & 0.4990 & 0.2554 & 0.49970613 & 0.00070613 & 0.25579957 & 0.00036147 \\
\hline 20 & 0.5265 & 0.4130 & 0.2174 & 0.41373367 & 0.00073367 & 0.21783078 & 0.00038628 \\
\hline 21 & 0.5398 & 0.3165 & 0.1708 & 0.31754621 & 0.00104621 & 0.17141144 & 0.00056474 \\
\hline 22 & 0.5521 & 0.2120 & 0.1170 & 0.21212300 & 0.00012300 & 0.11711311 & 0.00006791 \\
\hline 23 & 0.5633 & 0.1035 & 0.0583 & 0.10216328 & 0.00133672 & 0.05754857 & 0.00075298 \\
\hline 24 & 0.5736 & -0.0100 & -0.0057 & -0.00879175 & 0.00120825 & -0.00504295 & 0.00069305 \\
\hline 25 & 0.5833 & -0.1230 & -0.0717 & -0.12554343 & 0.00254343 & -0.07322949 & 0.00148359 \\
\hline 26 & 0.5900 & -0.2100 & -0.1239 & -0.20837159 & 0.00162841 & -0.12293924 & 0.00096076 \\
\hline Sum of IAE & & & & & 0.02127523 & & 0.00877664 \\
\hline
\end{tabular}

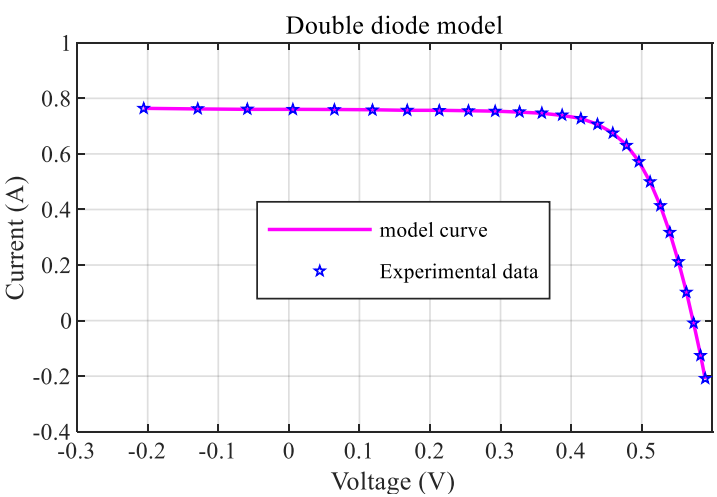

(a)

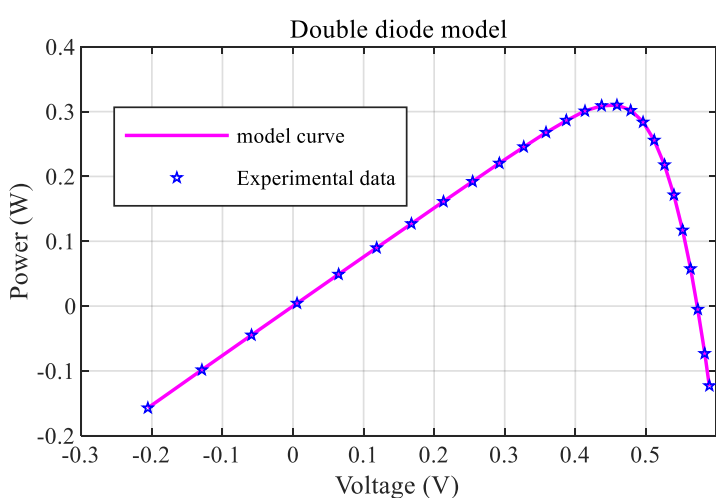

(b)

Fig. 9 Comparisons between experimental data and simulated data obtained by SCCSO on double diode model: (a) I-V characteristic, (b) P-V characteristic

Table 8 presents the five adjusted parameters and related RMSE values. Interestingly, SCCSO and PGJAYA obtain the best RMSE value (2.42507E-03) while the second-best RMSE value (2.42508E-03) is obtained by IBSO. As mentioned above, the 
slight difference in objective function has a significant impact on the accuracy of the adjusted parameters. Hence, SCCSO has strong competitiveness in the accuracy of parameters adjustment. Table 9 displays the IAE values of current and power, which are no more than 0.04892368 and 0.51688808 , respectively.

Furthermore, Fig. $10^{4}$ shows the I-V and P-V curves, and we can see that the simulated data are in great agreement with the experimental data within the whole voltage range.

Table 8 Comparisons of experimental results on PV module

\begin{tabular}{|c|c|c|c|c|c|c|}
\hline Algorithm & $I_{P H}(\mathrm{~A})$ & $I_{S D}(\mu \mathrm{A})$ & $R_{L}(\Omega)$ & $R_{S H}(\Omega)$ & $n$ & $R M S E$ \\
\hline SCCSO & 1.0305 & 3.48226 & 1.2013 & 981.9823 & 48.6428 & 2.42507E-03 \\
\hline $\mathrm{CSO}$ & 1.0301 & 3.74181 & 1.1937 & 1067.3017 & 48.9195 & $2.43329 \mathrm{E}-03$ \\
\hline $\mathrm{ABC}$ & 1.0296 & 3.70463 & 1.1955 & 1127.7167 & 48.8777 & $2.44438 \mathrm{E}-03$ \\
\hline MFO & 1.0293 & 4.32274 & 1.1786 & 1290.2168 & 49.4843 & $2.49818 \mathrm{E}-03$ \\
\hline IMFO & 1.0303 & 3.53721 & 1.1998 & 1009.1064 & 48.7025 & $2.42560 \mathrm{E}-03$ \\
\hline IJAYA & 1.0305 & 3.50358 & 1.2004 & 983.5366 & 48.6665 & $2.42525 \mathrm{E}-03$ \\
\hline PGJAYA & 1.0305 & 3.48261 & 1.2013 & 982.1342 & 48.6432 & $2.42507 \mathrm{E}-03$ \\
\hline IBSO & 1.0305 & 3.48318 & 1.2012 & 982.4548 & 48.6438 & 2.42508E-03 \\
\hline
\end{tabular}

Table 9 IAE of SCCSO on PV module

\begin{tabular}{|c|c|c|c|c|c|c|c|}
\hline \multirow{2}{*}{ Item } & \multicolumn{2}{|c|}{ Measured data } & \multirow{2}{*}{$\frac{\text { Calculated data }}{P(W)}$} & \multicolumn{2}{|c|}{ Simulated current data } & \multicolumn{2}{|c|}{ Simulated power data } \\
\hline & $U(V)$ & $I(A)$ & & $I_{\text {sim }}(A)$ & $I A E_{I}$ & $P_{\text {sim }}(W)$ & $I A E_{P}$ \\
\hline 1 & 0.1248 & 1.0315 & 0.1287 & 1.02911916 & 0.00238084 & 0.12843407 & 0.00029713 \\
\hline 2 & 1.8093 & 1.0300 & 1.8636 & 1.02738107 & 0.00261893 & 1.85884058 & 0.00473842 \\
\hline 3 & 3.3511 & 1.026 & 3.4382 & 1.02574180 & 0.00025820 & 3.43736334 & 0.00086526 \\
\hline 4 & 4.7622 & 1.0220 & 4.8670 & 1.02410715 & 0.00210715 & 4.87700309 & 0.01003469 \\
\hline 5 & 6.0538 & 1.0180 & 6.1628 & 1.02229180 & 0.00429180 & 6.18875013 & 0.02598173 \\
\hline 6 & 7.2364 & 1.0155 & 7.3486 & 1.01993068 & 0.00443068 & 7.38062638 & 0.03206218 \\
\hline 7 & 8.3189 & 1.0140 & 8.4354 & 1.01636311 & 0.00236311 & 8.45502304 & 0.01965844 \\
\hline 8 & 9.3097 & 1.0100 & 9.4028 & 1.01049615 & 0.00049615 & 9.40741602 & 0.00461902 \\
\hline 9 & 10.2163 & 1.0035 & 10.2521 & 1.00062897 & 0.00287103 & 10.22272575 & 0.02933130 \\
\hline 10 & 11.0449 & 0.9880 & 10.9124 & 0.98454838 & 0.00345162 & 10.87423839 & 0.03812281 \\
\hline 11 & 11.8018 & 0.9630 & 11.3651 & 0.95952168 & 0.00347832 & 11.32408292 & 0.04105048 \\
\hline 12 & 12.4929 & 0.9255 & 11.5622 & 0.92283882 & 0.00266118 & 11.52893307 & 0.03324588 \\
\hline 13 & 13.1231 & 0.8725 & 11.4499 & 0.87259966 & 0.00009966 & 11.45121264 & 0.00130789 \\
\hline 14 & 13.6983 & 0.8075 & 11.0614 & 0.80727426 & 0.00022574 & 11.05828505 & 0.00309220 \\
\hline 15 & 14.2221 & 0.7265 & 10.3324 & 0.72833648 & 0.00183648 & 10.35847423 & 0.02611858 \\
\hline 16 & 14.6995 & 0.6345 & 9.3268 & 0.63713800 & 0.00263800 & 9.36561003 & 0.03877728 \\
\hline 17 & 15.1346 & 0.5345 & 8.0894 & 0.53621306 & 0.00171306 & 8.11537023 & 0.02592653 \\
\hline 18 & 15.5311 & 0.4275 & 6.6395 & 0.42951132 & 0.00201132 & 6.67078334 & 0.03123809 \\
\hline 19 & 15.8929 & 0.3185 & 5.0619 & 0.31877448 & 0.00027448 & 5.06625098 & 0.00436233 \\
\hline 20 & 16.2229 & 0.2085 & 3.3825 & 0.20738951 & 0.00111049 & 3.36445923 & 0.01801542 \\
\hline 21 & 16.5241 & 0.1010 & 1.6689 & 0.09616717 & 0.00483283 & 1.58907596 & 0.07985814 \\
\hline 22 & 16.7987 & 0.0080 & 0.1344 & -0.00832539 & 0.00032539 & -0.13985567 & 0.00546607 \\
\hline 23 & 17.0499 & -0.1110 & -1.8925 & -0.11093648 & 0.00006352 & -1.89145594 & 0.00108296 \\
\hline 24 & 17.2793 & -0.2090 & -3.6114 & -0.20924727 & 0.00024727 & -3.61564628 & 0.00427258 \\
\hline 25 & 17.4885 & -0.3030 & -5.2990 & -0.30086359 & 0.00213641 & -5.26165284 & 0.03736266 \\
\hline Sum of IAE & & & & & 0.04892368 & & 0.51688808 \\
\hline
\end{tabular}

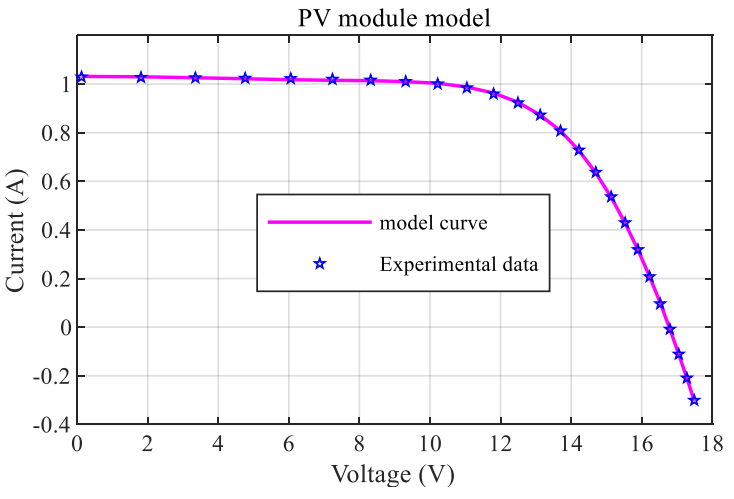

(a)

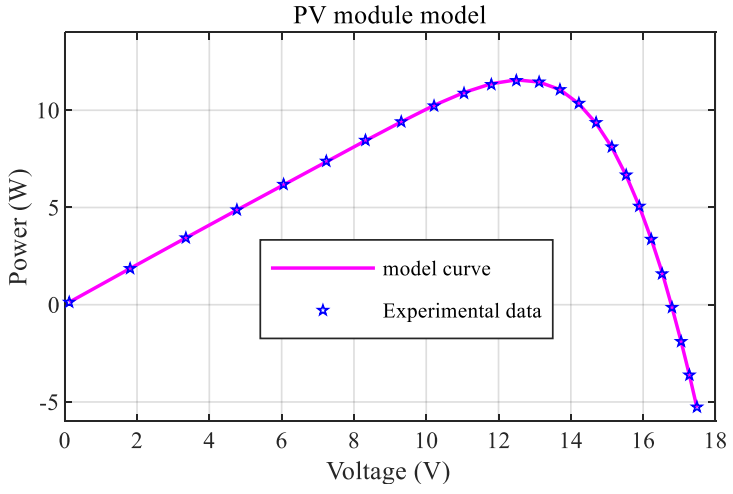

(b)

Fig. 10 Comparisons between experimental data and simulated data obtained by SCCSO on PV module: (a) I-V characteristic, (b) P-V characteristic

\subsection{Sensitivity analysis of crucial parameters}

As discussed in Section $4, r$ and $\mathfrak{I}$ are crucial to improve the performance of SCCSO. $r$ is the ratio of individuals in each sub-flock, and $\mathfrak{J}$ is the constant controlling when the chicks follow the rooster foraging. In this section, to investigate the sensitivity of $r$ and $\mathfrak{I}$, some experiments are conducted without changing other experimental settings. In previous experiments, $r$ is set to $1: 2: 1$ and $\mathfrak{J}$ is 0.4 . Here, different values are set for $r: 3: 14: 10,1: 1: 1$ and $1: 3: 1$, and different $\mathfrak{J}: 0.3$ and 0.5 . Table 
10 displays the experimental results of SCCSO using combinations of $r$ and $\mathfrak{J}$ for three models.

Table 10 Experimental results of SCCSO using combinations of $r$ and $\mathfrak{J}$ for three models

\begin{tabular}{|c|c|c|c|c|c|}
\hline \multirow{2}{*}{ Model } & \multirow{2}{*}{ Combination } & \multicolumn{4}{|c|}{ RMSE } \\
\hline & & Min & Mean & Max & SD \\
\hline \multirow{7}{*}{$\begin{array}{l}\text { Single diode } \\
\text { model }\end{array}$} & $\widetilde{J}=0.4, r=1: 2: 1$ & $9.86022 E-04$ & $9.86022 E-04$ & $9.86022 \mathrm{E}-04$ & 2.35724E-17 \\
\hline & $\mathfrak{J}=0.3, r=3: 14: 10$ & $9.86022 \mathrm{E}-04$ & $9.86022 E-04$ & $9.86022 \mathrm{E}-04$ & $2.85964 \mathrm{E}-17$ \\
\hline & $\mathfrak{J}=0.5, r=3: 14: 10$ & $9.86022 \mathrm{E}-04$ & $9.86022 E-04$ & $9.86022 E-04$ & $2.42036 \mathrm{E}-17$ \\
\hline & $\widetilde{J}=0.3, r=1: 1: 1$ & $9.86022 \mathrm{E}-04$ & $9.86022 E-04$ & $9.86022 \mathrm{E}-04$ & $2.68358 \mathrm{E}-17$ \\
\hline & $\widetilde{J}=0.5, \quad r=1: 1: 1$ & $9.86022 \mathrm{E}-04$ & $9.86022 E-04$ & $9.86022 \mathrm{E}-04$ & 2.38761E-17 \\
\hline & $\widetilde{J}=0.3, r=1: 3: 1$ & $9.86022 \mathrm{E}-04$ & $9.86022 E-04$ & $9.86022 \mathrm{E}-04$ & $2.41871 \mathrm{E}-17$ \\
\hline & $\mathfrak{J}=0.5, \quad r=1: 3: 1$ & $9.86022 \mathrm{E}-04$ & $9.86022 \mathrm{E}-04$ & $9.86022 \mathrm{E}-04$ & 2.93259E-17 \\
\hline \multirow{7}{*}{$\begin{array}{l}\text { Double diode } \\
\text { model }\end{array}$} & $\mathfrak{J}=0.4, \quad r=1: 2: 1$ & $9.82485 E-04$ & $9.83663 \mathrm{E}-04$ & $9.86090 \mathrm{E}-04$ & 1.41713E-06 \\
\hline & $\mathfrak{J}=0.3, r=3: 14: 10$ & $9.82485 \mathrm{E}-04$ & 9.87357E-04 & $1.02978 \mathrm{E}-03$ & $9.11867 \mathrm{E}-06$ \\
\hline & $\mathfrak{I}=0.5, \quad r=3: 14: 10$ & $9.82534 \mathrm{E}-04$ & $9.99856 \mathrm{E}-04$ & $9.86645 \mathrm{E}-04$ & 3.05001E-06 \\
\hline & $\Im=0.3, r=1: 1: 1$ & $9.82485 \mathrm{E}-04$ & $9.84143 \mathrm{E}-04$ & $9.91049 \mathrm{E}-04$ & $1.99448 \mathrm{E}-06$ \\
\hline & $\widetilde{J}=0.5, \quad r=1: 1: 1$ & $9.82485 E-04$ & $9.84068 E-04$ & $9.86422 \mathrm{E}-04$ & 1.50059E-06 \\
\hline & $\mathfrak{J}=0.3, r=1: 3: 1$ & $9.82485 E-04$ & $9.84295 \mathrm{E}-04$ & $9.86126 \mathrm{E}-04$ & $1.57948 \mathrm{E}-06$ \\
\hline & $\mathfrak{I}=0.5, \quad r=1: 3: 1$ & $9.82485 \mathrm{E}-04$ & $9.84417 \mathrm{E}-04$ & $9.92203 \mathrm{E}-04$ & 2.17523E-06 \\
\hline \multirow{7}{*}{$\begin{array}{l}\text { PV module } \\
\text { model }\end{array}$} & $\mathfrak{J}=0.4, \quad r=1: 2: 1$ & $2.42507 \mathrm{E}-03$ & $2.42507 \mathrm{E}-03$ & $2.42507 \mathrm{E}-03$ & $1.59503 \mathrm{E}-17$ \\
\hline & $\mathfrak{J}=0.3, r=3: 14: 10$ & $2.42507 \mathrm{E}-03$ & $2.42507 \mathrm{E}-03$ & $2.42507 \mathrm{E}-03$ & $1.95130 \mathrm{E}-17$ \\
\hline & $\Im=0.5, r=3: 14: 10$ & $2.42507 \mathrm{E}-03$ & $2.42507 \mathrm{E}-03$ & $2.42507 \mathrm{E}-03$ & $1.93792 \mathrm{E}-17$ \\
\hline & $\mathfrak{J}=0.3, r=1: 1: 1$ & $2.42507 \mathrm{E}-03$ & $2.42507 \mathrm{E}-03$ & $2.42507 \mathrm{E}-03$ & 1.65169E-17 \\
\hline & $\mathfrak{I}=0.5, \quad r=1: 1: 1$ & $2.42507 \mathrm{E}-03$ & $2.42507 \mathrm{E}-03$ & $2.42507 \mathrm{E}-03$ & $1.65594 \mathrm{E}-17$ \\
\hline & $\mathfrak{J}=0.3, \quad r=1: 3: 1$ & $2.42507 \mathrm{E}-03$ & $2.42507 \mathrm{E}-03$ & $2.42507 \mathrm{E}-03$ & $1.78661 \mathrm{E}-17$ \\
\hline & $\mathfrak{J}=0.5, r=1: 3: 1$ & $2.42507 \mathrm{E}-03$ & 2.42507E-03 & $2.42507 \mathrm{E}-03$ & $1.97725 \mathrm{E}-17$ \\
\hline
\end{tabular}

From Table 10, the variants all obtain the best results on the single diode model and PV module models, but only SCCSO achieves the best results on the double diode model with the best stability. Hence, by comparing the results with the combinations, we can conclude that the combination of $r=1: 2: 1$ and $\mathfrak{J}=0.4$ is a suitable choice for SCCSO.

\subsection{Authentication of strategy effectiveness}

To verify the effectiveness of the proposed strategies, it is essential to conduct several experiments. In this section, three variants are proposed based on SCCSO, which are SCCSO without the information-sharing strategy instead of the original rooster optimization equation in CSO (denoted as SCCSO-1), SCCSO without spiral motion trajectory equation instead of the original update equation of hen and chicks (denoted as SCCSO-2), and SCCSO without the self-adaptive-based chaotic disturbance mechanism (denoted as SCCSO-3). In the experiments, the parameter settings stay the same as mentioned. Table 11 presents the experimental results of SCCSO and its three variants on PV models in 30 independent runs. The results obtained by SCCSO performs better in all PV models than SCCSO-1. Although SCCSO-2 obtained the best results on the single diode model and PV module models, the results on the double diode model are not as good as the results obtained by SCCSO. As for SCCSO-3, it obtaines the best results on the single diode model, but the performance on the other two models are not as good as SCCSO. Comparisons indicate the extraordinary performance of SCCSO in robustness, accuracy, and avoiding local optimal. As a consequence, using a single strategy is insufficient to achieve satisfactory results, but combining all proposed strategies leads to the best results.

Table 11 Comparisons of SCCSO and its variants for three models

\begin{tabular}{cccccc}
\hline \multirow{2}{*}{ Model } & Algorithm & \multicolumn{3}{c}{ RMSE } \\
\cline { 3 - 6 } & & Min & Mean & Max & SD \\
\hline \multirow{3}{*}{$\begin{array}{c}\text { Single diode } \\
\text { model }\end{array}$} & SCCSO & $\mathbf{9 . 8 6 0 2 2 E - 0 4}$ & $\mathbf{9 . 8 6 0 2 2 E - 0 4}$ & $\mathbf{9 . 8 6 0 2 2 E - 0 4}$ & $\mathbf{2 . 3 5 7 2 4 E - 1 7}$ \\
& SCCSO-1 & $\mathbf{9 . 8 6 0 2 2 E - 0 4}$ & $\mathbf{9 . 8 6 7 1 1 E - 0 4}$ & $\mathbf{9 . 9 1 7 2 0 E - 0 4}$ & $1.59080 \mathrm{E}-06$ \\
& SCCSO-2 & $\mathbf{9 . 8 6 0 2 2 E - 0 4}$ & $\mathbf{9 . 8 6 0 2 2 E - 0 4}$ & $\mathbf{9 . 8 6 0 2 2 E - 0 4}$ & $1.40373 \mathrm{E}-11$ \\
& SCCSO-3 & $\mathbf{9 . 8 6 0 2 2 E - 0 4}$ & $\mathbf{9 . 8 6 0 2 2 E - 0 4}$ & $\mathbf{9 . 8 6 0 2 2 E - 0 4}$ & $\mathbf{2 . 6 0 8 5 6 E - 1 7}$ \\
\hline \multirow{3}{*}{$\begin{array}{c}\text { Double diode } \\
\text { model }\end{array}$} & SCCSO & $\mathbf{9 . 8 2 4 8 5 E - 0 4}$ & $\mathbf{9 . 8 3 6 6 3 E - 0 4}$ & $\mathbf{9 . 8 6 0 9 0 E - 0 4}$ & $\mathbf{1 . 4 1 7 1 3 E - 0 6}$ \\
& SCCSO-1 & $\mathbf{9 . 8 2 4 8 7 E - 0 4}$ & $\mathbf{9 . 8 6 0 8 1 E - 0 4}$ & $\mathbf{1 . 0 0 1 2 2 E - 0 3}$ & $\mathbf{3 . 9 3 8 8 9 E - 0 6}$ \\
& SCCSO-2 & $9.83591 \mathrm{E}-04$ & $9.87476 \mathrm{E}-04$ & $1.02355 \mathrm{E}-03$ & $7.34131 \mathrm{E}-06$ \\
PV module & SCCSO-3 & $1.08174 \mathrm{E}-03$ & $2.58761 \mathrm{E}-03$ & $6.72875 \mathrm{E}-03$ & $1.21375 \mathrm{E}-03$ \\
\hline model & SCCSO & $\mathbf{2 . 4 2 5 0 7 E - 0 3}$ & $\mathbf{2 . 4 2 5 0 7 E - 0 3}$ & $\mathbf{2 . 4 2 5 0 7 E - 0 3}$ & $\mathbf{1 . 5 9 5 0 3 E - 1 7}$ \\
& SCCSO-1 & $\mathbf{2 . 4 2 5 0 7 E - 0 3}$ & $\mathbf{2 . 0 5 4 6 9 E - 0 2}$ & $\mathbf{2 . 7 4 2 5 1 E - 0 1}$ & $6.89644 \mathrm{E}-02$ \\
& SCCSO-2 & $\mathbf{2 . 4 2 5 0 7 E - 0 3}$ & $\mathbf{2 . 4 2 5 0 7 E - 0 3}$ & $\mathbf{2 . 4 2 5 0 7 E - 0 3}$ & $\mathbf{2 . 6 7 8 0 3 E - 1 7}$ \\
\hline
\end{tabular}


The above experimental results incicate that compared with the advanced algorithms, SCCSO obtaines significant advances on robustness and accuracy. The reason is due to the three strategies we used in SCCSO, covering the information-sharing strategy, the spiral motion strategy and the self-adaptive-based chaotic disturbance mechanism. Specific discussion shows as follows:

(1) The information-sharing strategy is developed to overcome the shortness of low utilization of the information. In CSO, roosters only learn from a randomly-selected rooster so that the information of other roosters cannot be fully utilized. By the information-sharing strategy, the roosters get information from the leader rooster and another randomly-selected rooster. Then, the roosters update their positions in an optimal range in the chicken flock, thus improving the information utilization and ensuring the exploitation ability.

(2) The spiral motion strategy is designed to decrease the probability of falling into local optimum. In CSO, when the cock falls into the local optimum, the hens and the chicks are affected by the rooster and have a high probability of falling into the local optimum. By the spiral motion strategy, the searching range for hens and chicks is expanded, which is favorable to get rid of the local optimum and balance the early exploration and the later exploitation. Specifically, hens and chicks move to the roosters with the spiral trajectory, so the whole chicken flock can gather near roosters at a fast speed, accelerating the convergence speed. When at the later iteration, chicks have already gathered around the food. At this time, chicks follow their mothers to search for food benefits the exploitation ability.

(3) The self-adaptive-based chaotic disturbance mechanism is used to accelerate the convergence speed of the chicken flock. In $\mathrm{CSO}$, chicks neither learn from rooster when foraging nor use the latest information in the chicken flock. Thus, they may fail to concentrate near food effectively, degenerating the convergence speed of the chicken flock. By the self-adaptive-based chaotic disturbance mechanism, the worst chick moves to the position of the leader rooster derectly. After sorting fitness values of chickens, the new worst chick also moves to the new leader rooster, thus accelerating the convergence speed of the chicken flock without tripping into local optimum.

In summary, SCCSO is an effective choice to solve the problem of nonlinear multi-modal PV model parameter identification. However, according to the no free lunch theorem (Wolpert and Macreadym, 1997), SCCSO is not suitable for solving all optimization problems. When it is used to solve complex optimization problems with high dimensions, the results are not satisfied enough, and the population diversity needs to be improved. In the future, our work focus on rationally introducing evolutionary operators to increase the diversity of the chicken flock, improving the performance of SCCSO. It is intresting to use it for solving other multi-objective and constrained optimization problems in the power system, such as the fault ride-through improvement and the maximum power point tracking of speed wind generators (Qais et al. 2019b; Qais et al. 2020b).

\section{Conclusion}

It is crucial to determine accrurate and reliable PV model parameters for the evaluation and optimization of PV systems. However, the parameter identification problems of PV models have multimodal and nonlinear features so that most existing heuristic algorithms fail to obtain globally optimal solutions. To overcome this problem, this paper proposed a spiral-based chaos chicken swarm optimization algorithm (SCCSO) to identify the parameters of PV models. The experimental results show that SCCSO performs better in robustness and accuracy than other advanced heuristic algorithms on the single diode, double diode, and PV module models. The reasons behind the fact are: i) SCCSO develops the information-sharing strategy to provide the latest information of roosters for exploring local optimal solutions, which is beneficial to improve the exploitation ability; ii) SCCSO develops the spiral motion strategy to expand the searching range of hens and chicks, improving the exploration ability; iii) SCCSO introduces the self-adaptive-based chaotic disturbance mechanism to update the worst chick based on the global optimal solution, improving the convergence speed of the chicken flock; iv) SCCSO rationally combines the above three strategies and thus achieves satisfactory results. Thus, SCCSO is a promising candidate technique for the parameters identification problems of PV models.

\section{References}

A GX, A JZ, B DS, C LZ, A XYJEC. (2020). Parameter extraction of solar photovoltaic models with an either-or teaching learning based algorithm. Energy Conversion and Management 224,113395 doi:10.1016/j.enconman.2020.113395.

Abdel-Basset M, El-Shahat D, Chakrabortty RK, Ryan M (2021a). Parameter estimation of photovoltaic models using an improved marine predators algorithm. Energy Conversion and Management 227, doi:10.1016/j.enconman.2020.113491.

Abdel-Basset M, Mohamed R, Chakrabortty RK, Sallam K, Ryan MJ (2021b). An efficient teaching-learning-based optimization algorithm for parameters identification of photovoltaic models: Analysis and validations. Energy Conversion and Management 227, doi:10.1016/j.enconman.2020.113614.

Ahmed K, Hassanien AE, Ezzat E, Bhattacharyya S (2018). Swarming Behaviors of Chicken for Predicting Posts on Facebook Branding Pages. The International Conference on Advanced Machine Learning Technologies and Applications (AMLTA2018).

Al Shayokh M, Shin SY (2017). Bio Inspired Distributed WSN Localization Based on Chicken Swarm Optimization Wireless Personal Communications. Wireless Personal Communications 97:5691-5706, doi:10.1007/s11277-017-4803-1.

Alcalá-Fdez J et al. (2008). KEEL: a software tool to assess evolutionary algorithms for data mining problems. Soft Computing 13:307-318, doi:10.1007/s00500008-0323-y.

Allam D, Yousri DA, Eteiba MB (2016). Parameters extraction of the three diode model for the multi-crystalline solar cell/module using Moth-Flame Optimization Algorithm. Energy Conversion and Management 123:535-548, doi:10.1016/j.enconman.2016.06.052.

Aly SP, Ahzi S, Barth NJAE (2019). An adaptive modelling technique for parameters extraction of photovoltaic devices under varying sunlight and temperature conditions. Applied Energy 236:728-742, doi:.10.1016/j.apenergy.2018.12.036.

Chen X, Xu B, Mei C, Ding Y, Li K (2018). Teaching-learning-based artificial bee colony for solar photovoltaic parameter estimation. Applied Energy 212:15781588, doi:10.1016/j.apenergy.2017.12.115.

Chen X, Yu K, Du W, Zhao W, Liu G (2016). Parameters identification of solar cell models using generalized oppositional teaching learning based optimization .Energy 99:170-180, doi:10.1016/j.energy.2016.01.052.

Coelho LdS, Mariani VC (2008). Use of chaotic sequences in a biologically inspired algorithm for engineering design optimization. Expert Systems with Applications 34:1905-1913, doi:10.1016/j.eswa.2007.02.002. 
Diantoro M, Suprayogi T, Hidayat A, Taufiq A, Fuad A, Suryana R (2018). Shockley's Equation Fit Analyses for Solar Cell Parameters from I-V Curves. International Journal of Photoenergy 2018:1-7, doi:10.1155/2018/9214820.

Easwarakhanthan T, Bottin J, Bouhouch I, Boutrit C (1986). Nonlinear Minimization Algorithm for Determining the Solar Cell Parameters with Microcomputers International Journal of. Solar Energy 4:1-12, doi:10.1080/01425918608909835.

Elsheikh AH, Sharshir SW, Abd Elaziz M, Kabeel AE, Wang G, Zhang H (2019). Modeling of solar energy systems using artificial neural network: A comprehensive review. Solar Energy 180:622-639, doi:10.1016/j.solener.2019.01.037.

Eseye AT, Zhang J, Zheng DJRE (2017). Short-term photovoltaic solar power forecasting using a hybrid Wavelet-PSO-SVM model based on SCADA and Meteorological information. Renewable Energy 118:357-367, doi:10.1016/j.renene.2017.11.011.

Gao X, Cui Y, Hu J, Xu G, Wang Z, Qu J, Wang H (2018). Parameter extraction of solar cell models using improved shuffled complex evolution algorithm. Energy Conversion and Management 157:460-479, doi:10.1016/j.enconman.2017.12.033.

Gong L, Cao W, Zhao J. An improved PSO algorithm for high accurate parameter identification of PV model. In: International Conference on Environment and Electrical EngineeringIndustrial and Commercial Power Systems Europe.

Gude S, Jana KC (2020). Parameter extraction of photovoltaic cell using an improved cuckoo search optimization. Solar Energy 204:280-293, doi:10.1016/j.solener.2020.04.036.

Harrou F, Sun Y, Taghezouit B, Saidi A, Hamlati M-E (2018). Reliable fault detection and diagnosis of photovoltaic systems based on statistical monitoring approaches. Renewable Energy 116:22-37, doi:10.1016/j.renene.2017.09.048

Ismail FH, Houssein EH, Hassanien AE (2019). Chaotic Bird Swarm Optimization Algorithm. In: Hassanien AE, Tolba MF, Shaalan K, Azar AT (eds) Proceedings of the International Conference on Advanced Intelligent Systems and Informatics 2018, vol 845. Advances in Intelligent Systems and Computing. pp 294-303. doi:10.1007/978-3-319-99010-1 27.

Jiao S et al. (2020). Orthogonally adapted Harris hawks optimization for parameter estimation of photovoltaic models. Energy 203, doi:10.1016/j.energy.2020.117804.

Jordehi AR (2018). Enhanced leader particle swarm optimisation (ELPSO): An efficient algorithm for parameter estimation of photovoltaic (PV) cells and modules. Solar Energy 159:78-87, doi:10.1016/j.solener.2017.10.063.

Karaboga D, Ozturk C (2011). A novel clustering approach: Artificial Bee Colony (ABC) algorithm. Applied Soft Computing 11:652-657, doi:10.1016/j.asoc.2009.12.025.

Li S, Gu Q, Gong W, Ning BJEC, Management (2020). An enhanced adaptive differential evolution algorithm for parameter extraction of photovoltaic models. Energy Conversion and Management 205:112443-, doi:10.1016/j.enconman.2019.112443.

Liu Y et al. (2020). Horizontal and vertical crossover of Harris hawk optimizer with Nelder-Mead simplex for parameter estimation of photovoltaic models. Energy Conversion and Management 223, doi:10.1016/j.enconman.2020.113211.

Lu H, Wang X, Fei Z, Qiu MJMPiE (2014). The Effects of Using Chaotic Map on Improving the Performance of Multiobjective Evolutionary Algorithms Mathematical Problems in Engineering 2014:1-16, doi:10.1155/2014/924652.

Manel M, Anis S, Faouzi MMJEC, Management (2018). Particle swarm optimisation with adaptive mutation strategy for photovoltaic solar cell/module parameter extraction. Energy Conversion and Management 175:151-163, doi:10.1016/j.enconman.2018.08.081.

Meng X, Liu Y, Gao X, Zhang H (2014). A New Bio-inspired Algorithm: Chicken Swarm Optimization. In: Tan Y, Shi Y, Coello CAC (eds) Advances in Swarm Intelligence, Pt1, vol 8794. Lecture Notes in Computer Science. pp 86-94

Mirjalili SJK-BS (2015). Moth-flame optimization algorithm: A novel nature-inspired heuristic paradigm. Knowledge-Based Systems 89:228-249, doi:10.1016 / j.knosys.2015.07.006.

Moldovan D, Chifu V, Pop C, Cioara T, Anghel I, Salomie I (2018). Chicken Swarm Optimization and Deep Learning for Manufacturing Processes. In: Gasner P, Cebuc E (eds) 2018 17th Roedunet Ieee International Conference: Networking in Education and Research. RoEduNet International Conference.

Mu Y, Zhang L, Chen X, Gao X, Ieee (2016). Optimal Trajectory Planning for Robotic Manipulators Using Chicken Swarm Optimization. In: 2016 8th International Conference on Intelligent Human-Machine Systems and Cybernetics. International Conference on Intelligent Human-Machine Systems and Cybernetics. pp 369-373. doi:10.1109/ihmsc.2016.107.

Niu Q, Zhang H, Li K (2014). An improved TLBO with elite strategy for parameters identification of PEM fuel cell and solar cell models. International Journal of Hydrogen Energy 39:3837-3854, doi:10.1016/j.ijhydene.2013.12.110.

Oliv D, Mohamed AEA, Hassanien AEJAE (2017). Parameter estimation of photovoltaic cells using an improved chaotic whale optimization algorithm. Applied Energy 200:141-154, doi: 10.1016/j.apenergy.2017.05.029.

Qais M, Hasanien HM, Alghuwainem S, Nouh ASJE (2019a). Coyote Optimization Algorithm for Parameters Extraction of Three-diode Photovoltaic Models of Photovoltaic Modules. Energy 187:116001, doi:10.1016/j.energy.2019.116001.

Qais MH, Hasanien HM, Alghuwainem S (2019b). Enhanced salp swarm algorithm: Application to variable speed wind generators. Engineering Applications of Artificial Intelligence 80:82-96, doi:10.1016/j.engappai.2019.01.011.

Qais MH, Hasanien HM, Alghuwainem S (2020a). Parameters extraction of three-diode photovoltaic model using computation and Harris Hawks optimization. Energy 195, doi:10.1016/j.energy.2020.117040.

Qais MH, Hasanien HM, Alghuwainem S (2020b). Whale optimization algorithm-based Sugeno fuzzy logic controller for fault ride-through improvement of gridconnected variable speed wind generators. Engineering Applications of Artificial Intelligence 87, doi:10.1016/j.engappai.2019.103328.

Ridha HM, Heidari AA, Wang M, Chen H (2020). Boosted mutation-based Harris hawks optimizer for parameters identification of single-diode solar cell models. Energy Conversion and Management 209, doi:10.1016/j.enconman.2020.112660.

Sanchari D, Kari T, Karuna K, Pinakeshwar MJE (2018). Impact of Electric Vehicle Charging Station Load on Distribution Network. Energies 11:178, doi:10.3390/en11010178.

Sheng H, Li C, Wang H, Yan Z, Xiong Y, Cao Z, Kuang Q (2019). Parameters Extraction of Photovoltaic Models Using an Improved Moth-Flame Optimization. Energies 12, doi:10.3390/en12183527.

Taie SA, Ghonaim W, Ieee (2017). Title CSO-Based Algorithm with Support Vector Machine for brain tumor's disease Diagnosis. In: 2017 Ieee International Conference on Pervasive Computing and Communications Workshops. International Conference on Pervasive Computing and Communications.

Venkata Rao R (2016) Jaya: A simple and new optimization algorithm for solving constrained and unconstrained optimization problems. International Journal of Industrial Engineering Computations:19-34, doi:10.5267/j.ijiec.2015.8.004

Wu Y, Chen R, Li C, Zhang L, Cui Z (2020). Hybrid Symbiotic Differential Evolution Moth-Flame Optimization Algorithm for Estimating Parameters of Photovoltaic Models. Ieee Access 8:156328-156346, doi:10.1109/access.2020.3005711

Xiong G, Zhang J, Shi D, Zhu L, Yao GJRS (2019). Modified Search Strategies Assisted Crossover Whale Optimization Algorithm with Selection Operator for Parameter Extraction of Solar Photovoltaic Models. Remote Sensing 11:2795, doi:10.3390/rs11232795.

Yan Z, Li C, Song Z, Xiong L, Luo C (2019). An Improved Brain Storming Optimization Algorithm for Estimating Parameters of Photovoltaic Models. Ieee Access 7:77629-77641, doi:10.1109/access.2019.2922327.

Yu K, Chen X, Wang X, Wang Z (2017a). Parameters identification of photovoltaic models using self-adaptive teaching-learning-based optimization. Energy Conversion and Management 145:233-246, doi:10.1016/j.enconman.2017.04.054.

Yu K, Liang JJ, Qu BY, Chen X, Wang H (2017b). Parameters identification of photovoltaic models using an improved JAYA optimization algorithm. Energy Conversion and Management 150:742-753, doi:10.1016/j.enconman.2017.08.063.

Yu K, Liang JJ, Qu BY, Cheng Z, Wang H (2018). Multiple learning backtracking search algorithm for estimating parameters of photovoltaic models. Applied Energy 226:408-422, doi:10.1016/j.apenergy.2018.06.010.

Yu K, Qu B, Yue C, Ge S, Chen X, Liang J (2019). A performance-guided JAYA algorithm for parameters identification of photovoltaic cell and module. Applied Energy 237:241-257, doi:10.1016/j.apenergy.2019.01.008.

Yu X, Xie J, Jiang R, Zuo G, Liang J (2020). Assessment of water resource carrying capacity based on the chicken swarm optimization-projection pursuit model. Arabian Journal of Geosciences 13, doi:10.1007/s12517-019-5010-z. 
Zaimi M, Achouby HE, Ibral A, Assaid EMJSE (2019). Determining combined effects of solar radiation and panel junction temperature on all model-parameters to forecast peak power and photovoltaic yield of solar panel under non-standard conditions. Solar Energy 191:341-359, doi:10.1016/j.solener.2019.09.007.

Zhang J, Liu Y, Li Y, Ding K, Wu JJEC (2020a). A reinforcement learning based approach for on-line adaptive parameter extraction of photovoltaic array models. Energy Conversion and Management 214:112875, doi:10.1016/j.enconman.2020.112875.

Zhang Y, Ma M, Jin ZJE (2020b). Comprehensive learning Jaya algorithm for parameter extraction of photovoltaic models. Energy 211, doi:10.1016/j.energy.2020.118644. 
Figures

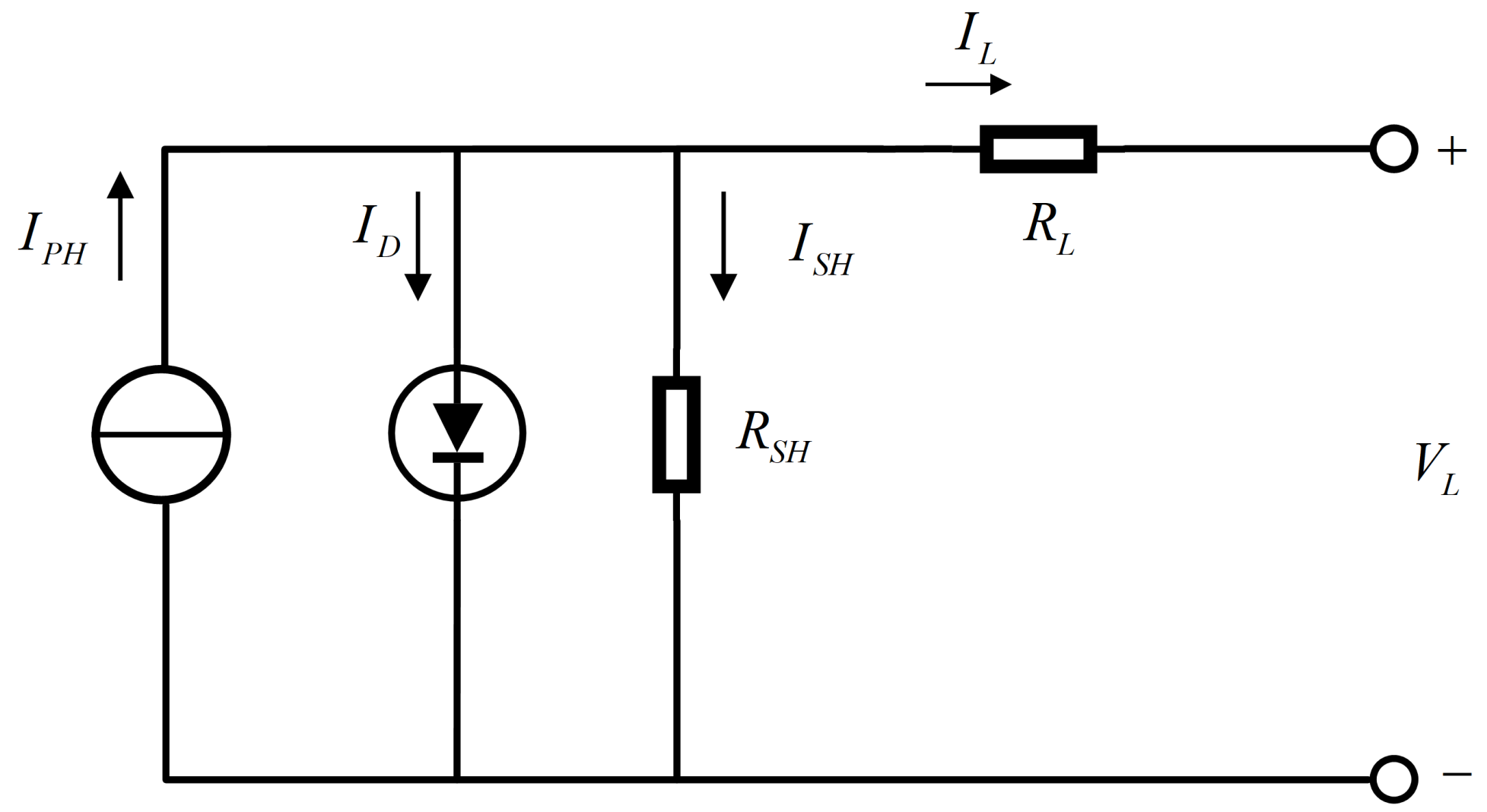

Figure 1

Equivalent circuit of the single diode model

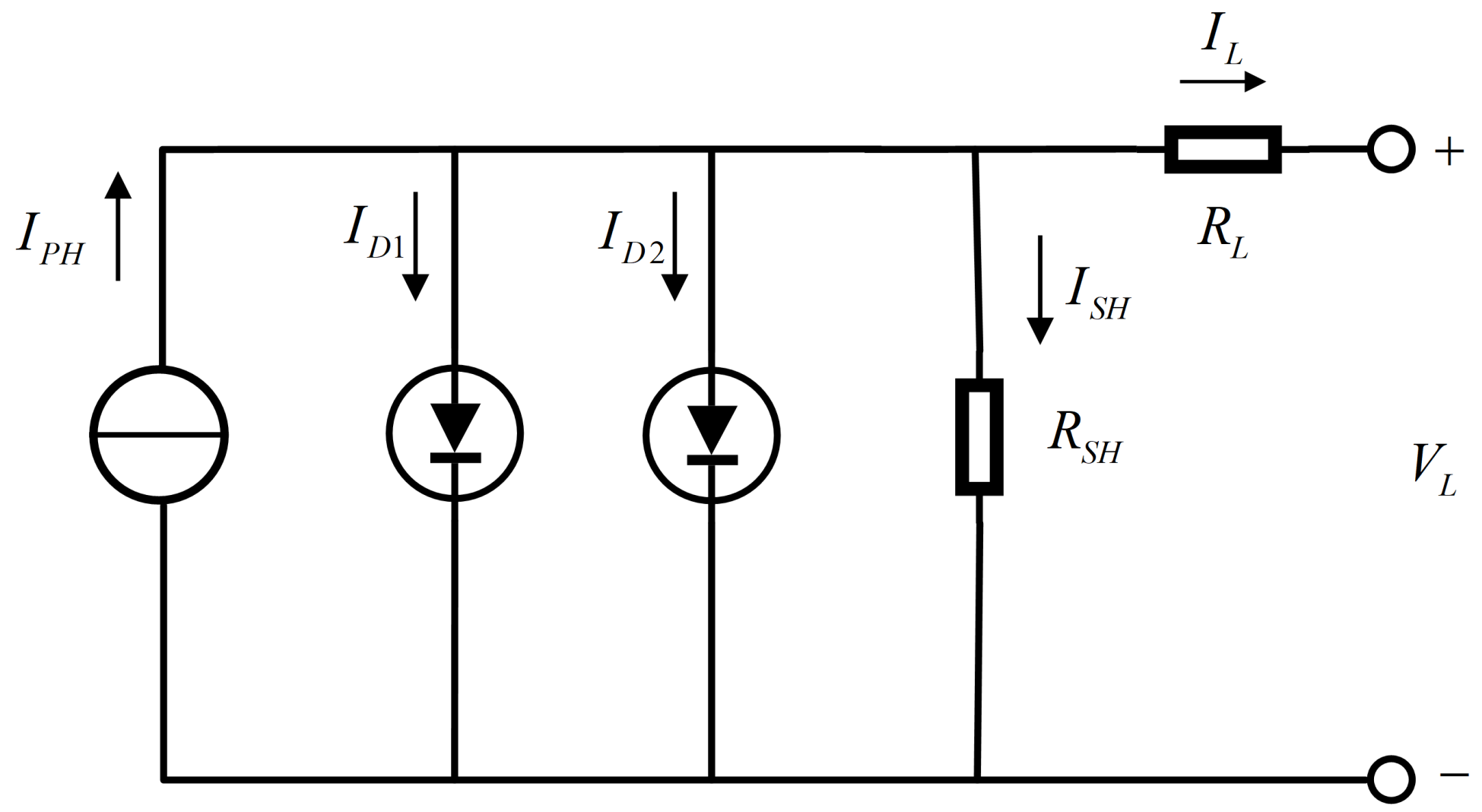


Figure 2

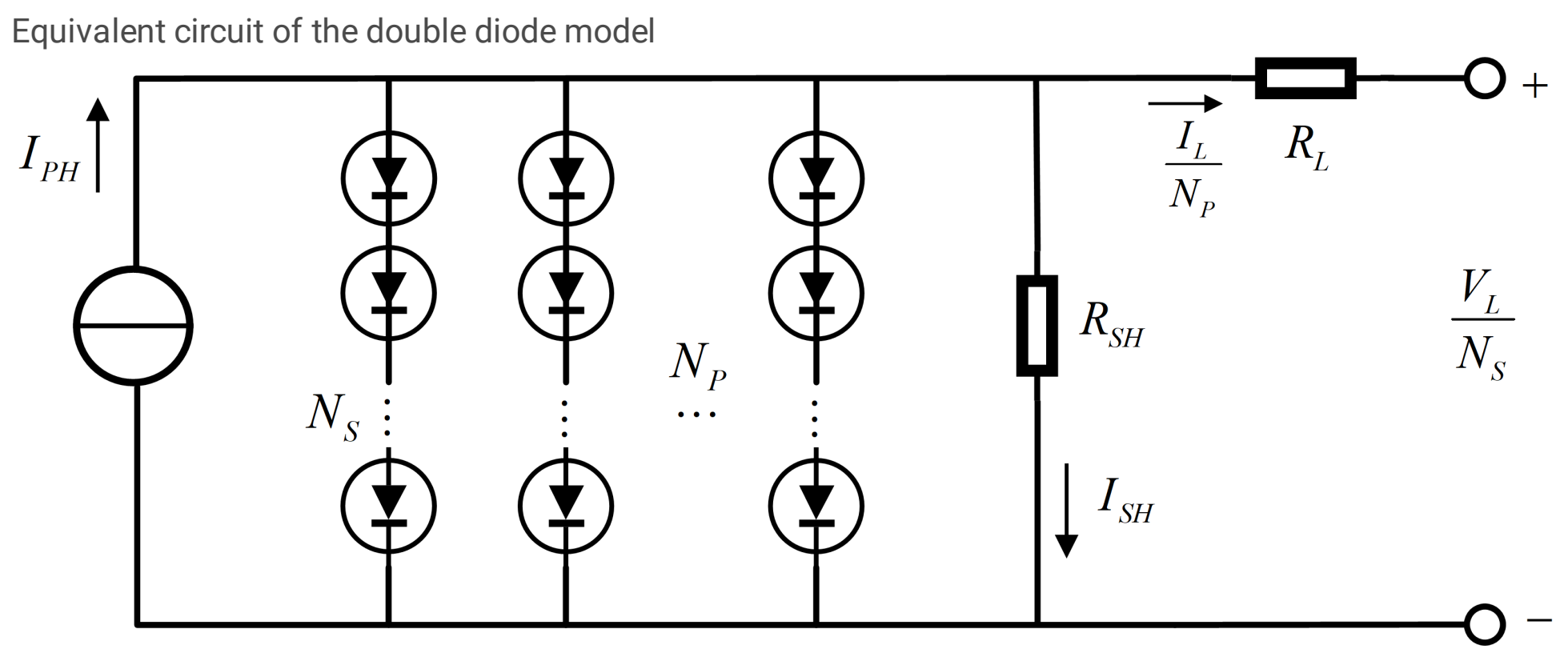

Figure 3

Equivalent circuit of the PV module model 


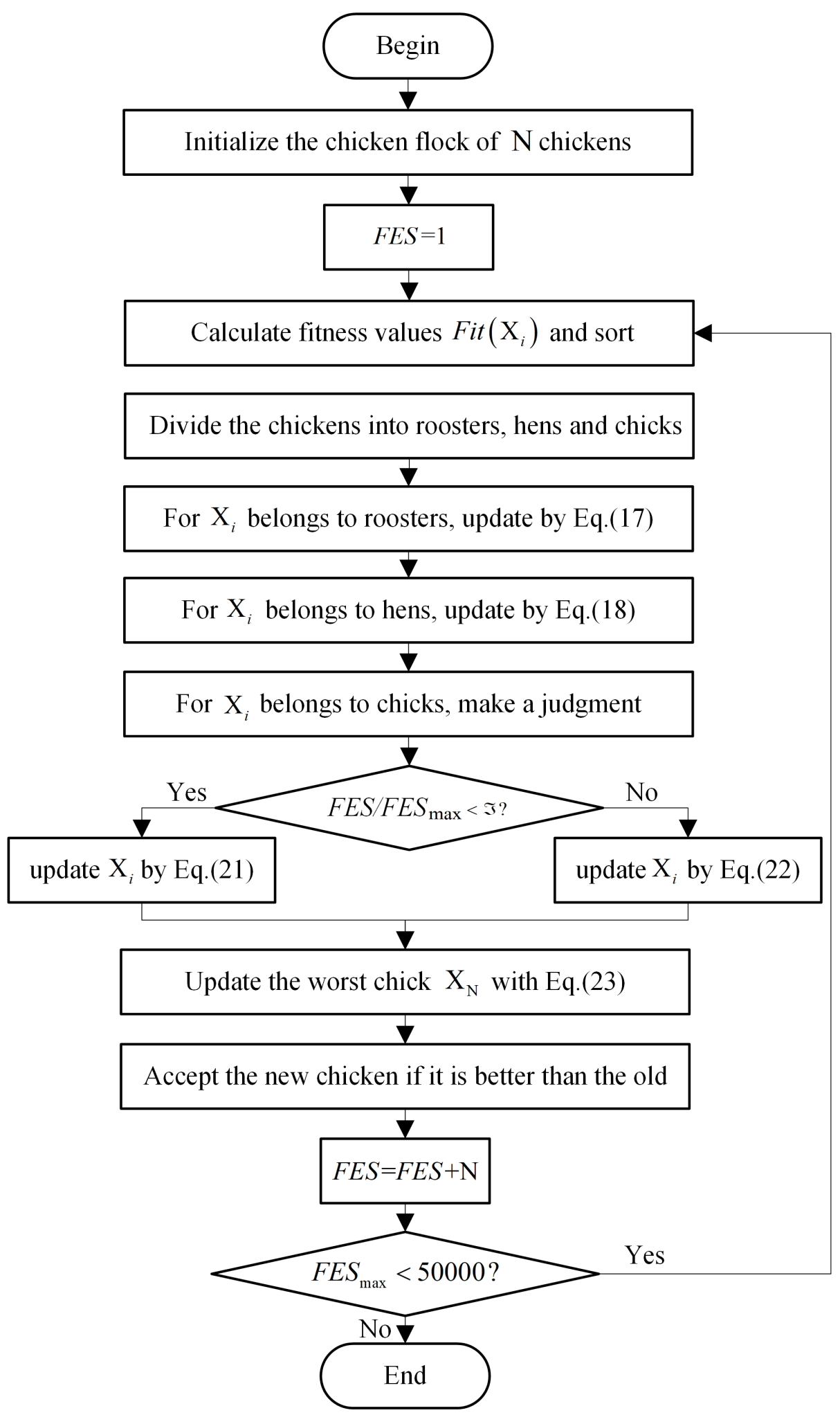

Figure 4

Flow chart of SCCSO 


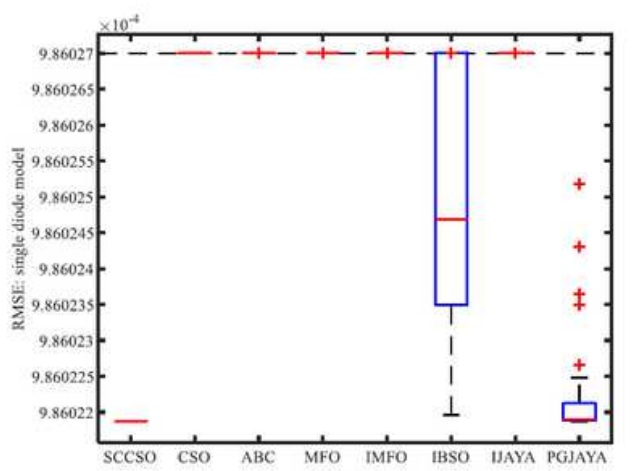

(a)

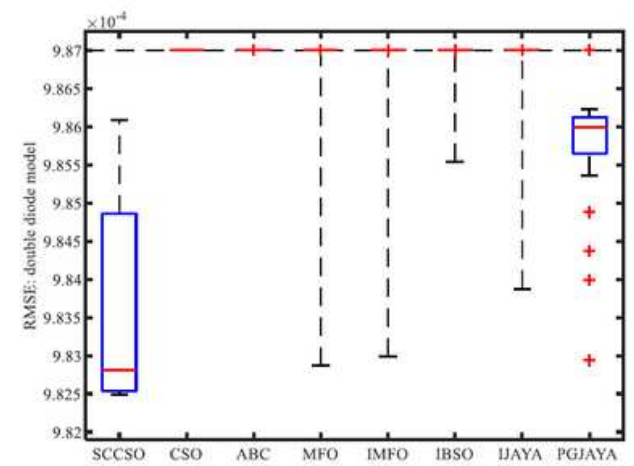

(b)

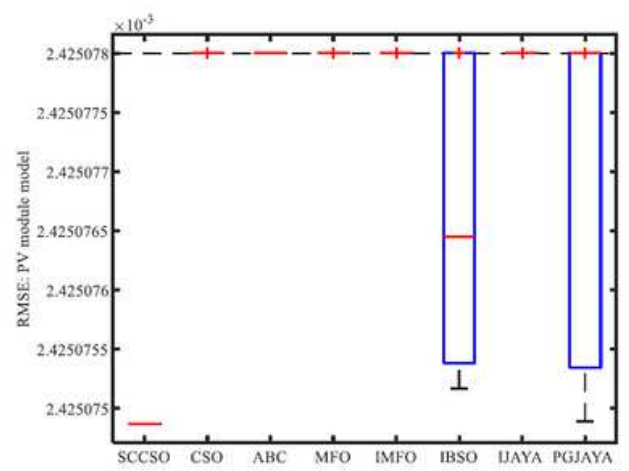

(c)

Figure 5

Best RMSE boxplot in 30 runs of different algorithms for: (a) single diode model, (b) double diode model, (c) PV module model

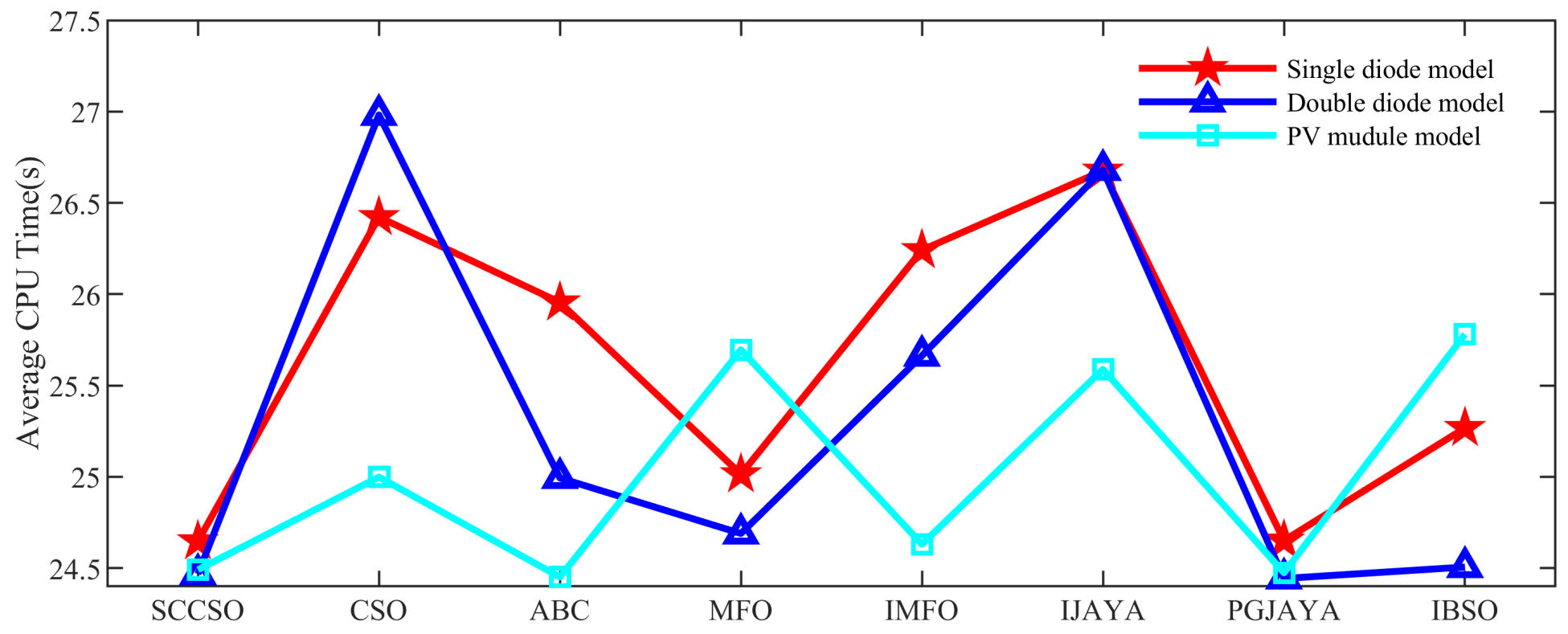

Figure 6

The average CPU time of different algorithms for three models 


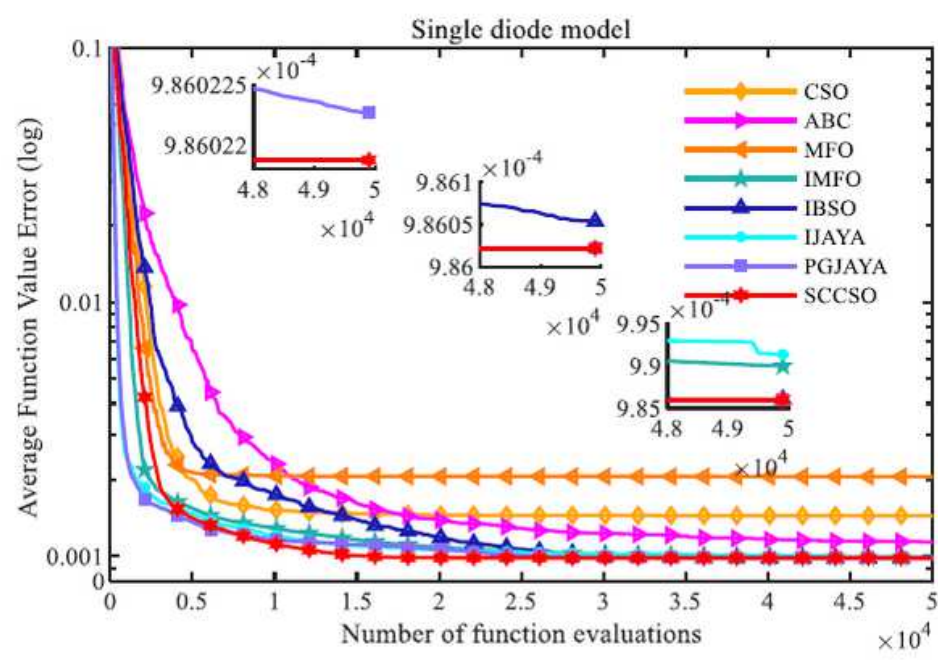

(a)

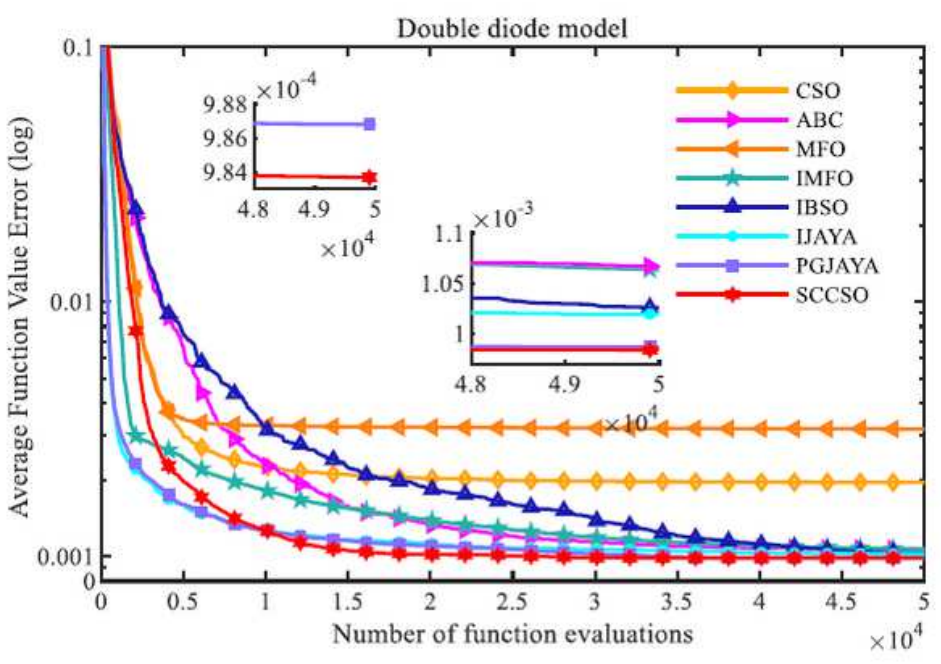

(b)

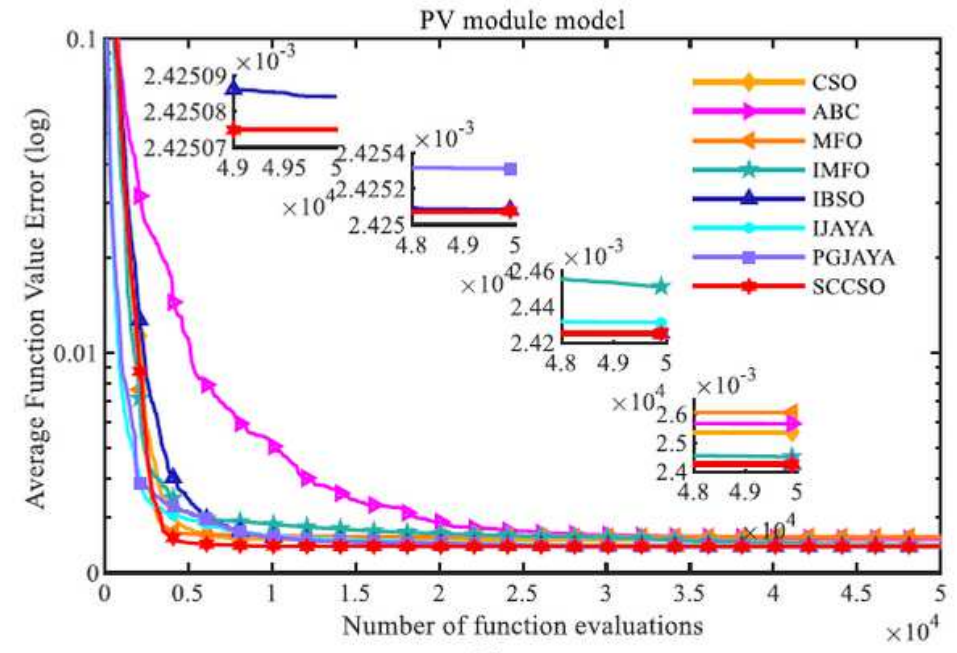

(c)

\section{Figure 7}

Convergence curves of SCCSO and compared algorithms for: (a) single diode model, (b) double diode model, (c) PV module model

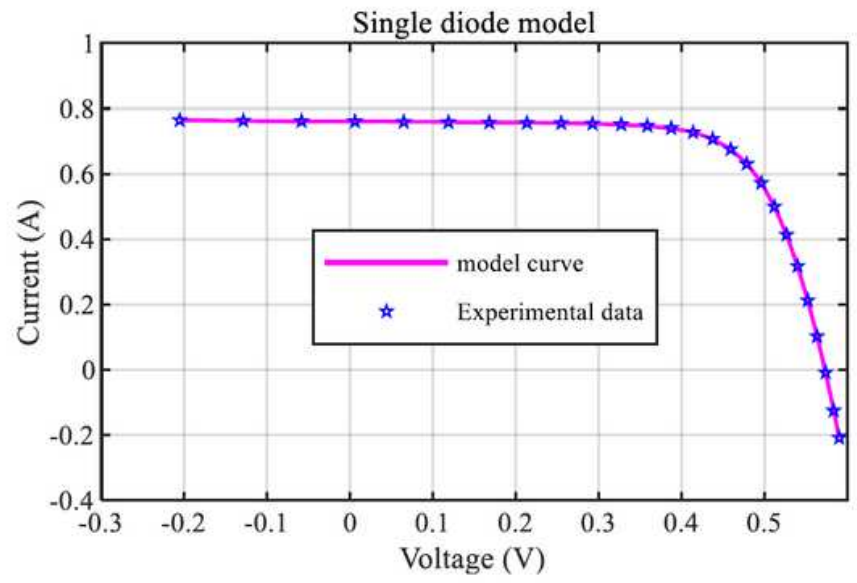

(a)

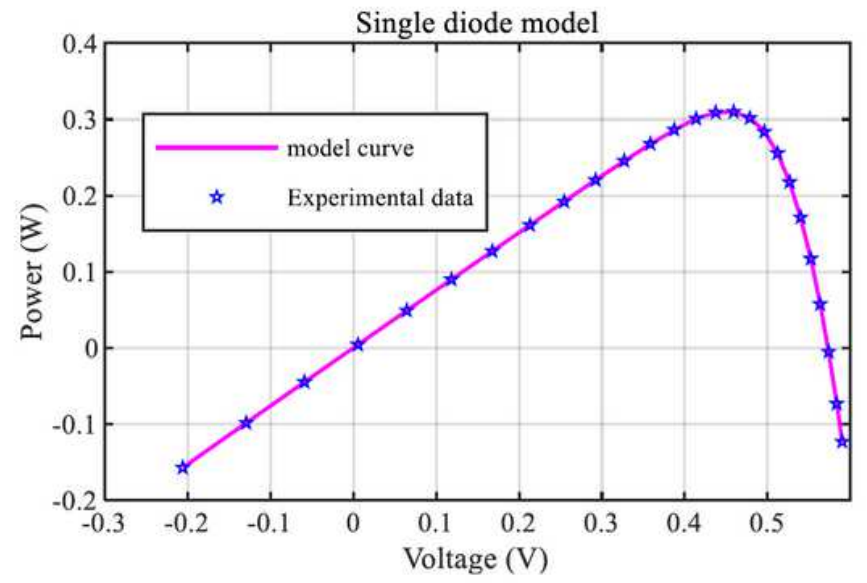

(b) 
Comparisons between experimental data and simulated data obtained by SCCSO on single diode model: (a) I-V characteristic, (b) P-V characteristic

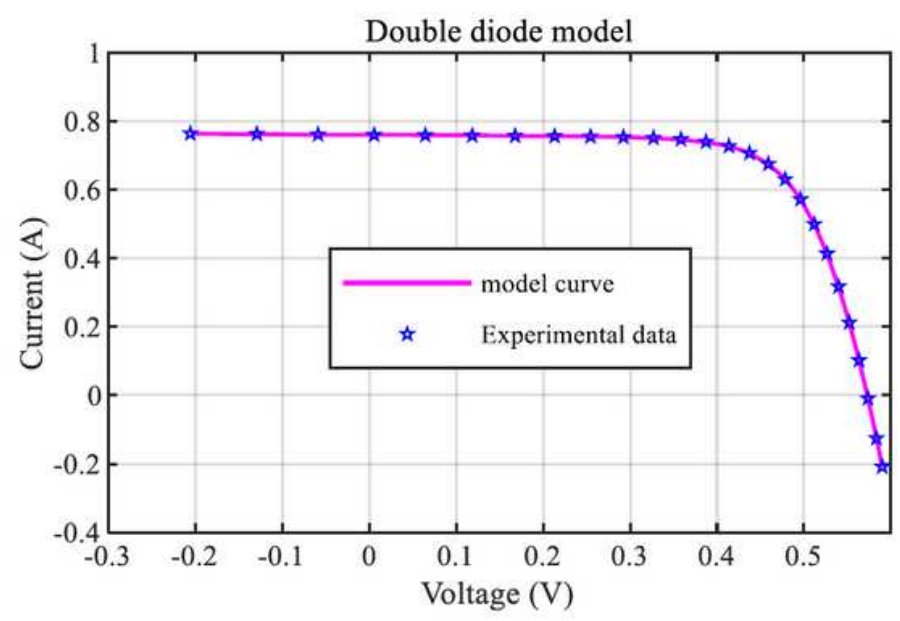

(a)

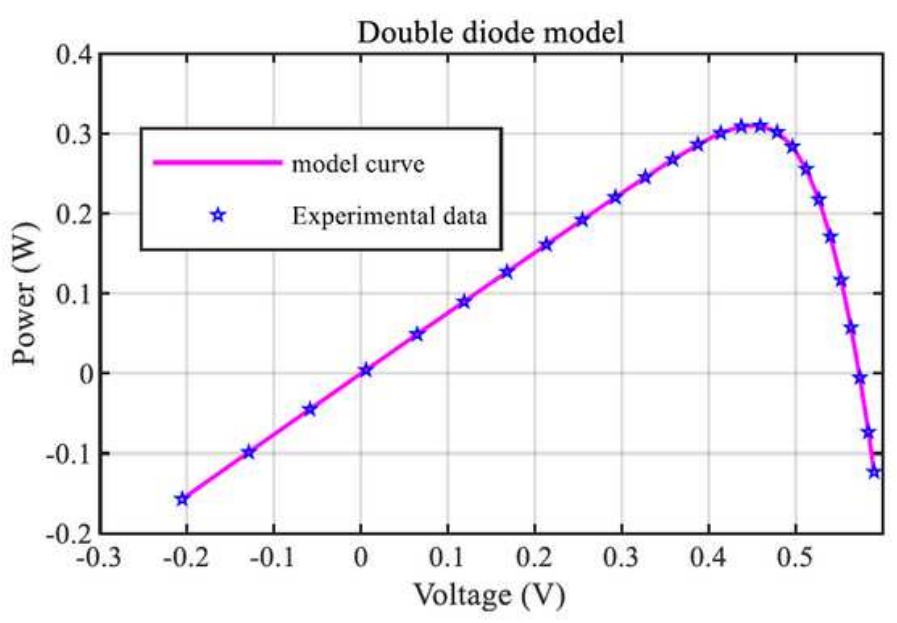

(b)

\section{Figure 9}

Comparisons between experimental data and simulated data obtained by SCCSO on double diode model: (a) I-V characteristic, (b) P-V characteristic

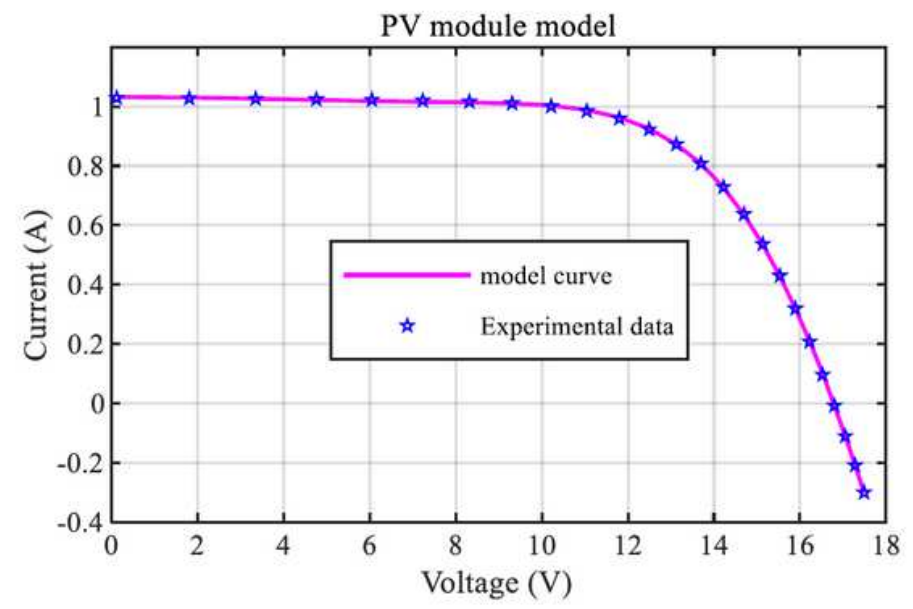

(a)

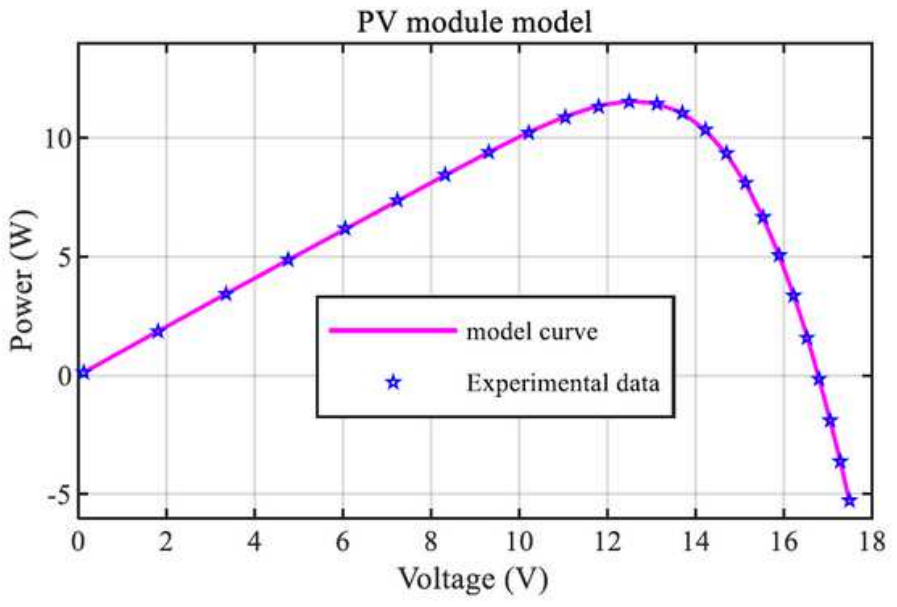

(b)

\section{Figure 10}

Comparisons between experimental data and simulated data obtained by SCCSO on PV module: (a) I-V characteristic, (b) P-V characteristic 